\title{
An Algorithm for Clustering Input Variables in a Fuzzy Model in a FLC Process
}

\author{
Nenad Stojanovic \\ Faculty of Agriculture, University of Banja Luka, Banja Luka, Bosnia and Herzegovina \\ Email address: \\ nenad.stojanovic@agro.unibl.org

\section{To cite this article:} \\ Nenad Stojanovic. An Algorithm for Clustering Input Variables in a Fuzzy Model in a FLC Process. International Journal of Management \\ and Fuzzy Systems. Vol. 6, No. 2, 2020, pp. 29-46. doi: 10.11648/j.ijmfs.20200602.12
}

Received: September 18, 2020; Accepted: October 6, 2020; Published: October 13, 2020

\begin{abstract}
The input and output variables in fuzzy systems are linguistic variables. The base of the fuzzy rule represents the central part of a fuzzy controller, and the fuzzy rule represents its basic part, and it has the following form: "if R then P", where $\mathrm{R}$ and $\mathrm{P}$ represent the fuzzy relation, i.e. the proposition. Complex systems described by fuzzy relations generate a large number of inference rules. Grouping the states into clusters on the basis of which we make conclusions about the value of the output variable is performed by an expert based on his or her experience and knowledge. Ideally, the number of clusters should correspond to the number of attributes by which the value of the output variable is classified, which, in reality is not the case. In the absence of experts, we perform grouping on the basis of some of the criteria. One way of grouping descriptive states into clusters is presented in this paper. It presents a construction of the method of grouping descriptive states of fuzzy models, with the aim of drawing conclusions about the value of the output variable described by a given state. The presented method of grouping descriptive states is based on defined characteristic values associated with fuzzy numbers by which the input variables of the model are evaluated. They represent the basis for defining the characteristic value of the descriptive state of the output variable of a fuzzy model. For the presented method, a mathematical logical argumentation of the application is given, as an algorithm for the application of the constructed method. The application of the algorithm is demonstrated in measuring the economic dimension of the sustainability of tourism development, measured by comparative evaluation indicators.
\end{abstract}

Keywords: Data Clustering, Reduction of Inference Rules, Algorithms, Mathematical Modeling, FLC Processes

\section{Introduction}

The meaning of a term is specified by the use of the attributes. For example, the seasonality of a visit can be high or low. Obviously, no definite value can be set in which the benefit ceases to be low. and becomes high. The boundary between these two attributes is elastic and mainly depends on a personal assessment and the circumstances in which the term is observed $[14,15]$. If we want a more precise specification of this term, then we can say, for example, that the loyalty is: very low., low, medium, high, very high. Obviously a larger number of attributes contributes to the linguistic specification of the term, but the problem of limits still remains open. Fuzzy sets can play a role of elastic limits between individual attributes. We associate a fuzzy set to each attribute individually and assign it a domain that has a clear semantic meaning. Generally, we can say that a family of fuzzy sets $\mathcal{A}=\left\{A_{1}, A_{2}, A_{3}, \ldots, A_{n}\right\}$ represents a framework of cognition of term $X$, if each element of term $X$ is associated with at least one fuzzy set with a non-zero degree of affiliation.

In the theory of fuzzy sets, a characteristic function, $\chi_{A}(x)$, affiliation of element $\mathrm{x}$ to set $\mathrm{A}$,

$$
\chi_{A}(x)= \begin{cases}1 & , x \in A \\ 0 & , x \notin A\end{cases}
$$

is generalized by a function of affiliation, i.e. $\mu_{A}$. The degree of affiliation of element $x$ to a fuzzy set is given by the real value from interval $[0,1]$, i.e. $\mu_{A}(x): A \rightarrow[0,1]$. Fuzzy set $A$ is completely determined with the set of ordered pairs: $[0,1]$, i.e.

$$
A=\left\{\left(x, \mu_{A}(x)\right) \mid x \in U, \mu_{A}(x) \in[0,1]\right\},
$$

where $\mu_{A}(x)$ is the degree of affiliation of element $x$ to set $A$, and $U$ is a universal set. If $\mu_{A}(x)$ is greater, then there is so much more truth in the claim that element $x$ belongs to set $A$. 
Fuzzy sets written in a triangular form are the most practical and most commonly used forms of the function of affiliation, Figure (1). A triangular fuzzy set is written shorter in the following form: $A=\operatorname{trim} f[l, m, n]$, and a function of affiliation $\mu_{A}(x): U \rightarrow[0,1]$ is given by the following equations

$$
\mu_{A}(x)=\left\{\begin{array}{ll}
0 & , n<x<l \\
\frac{x-l}{m-l} & , l \leq x \leq m \\
\frac{n-x}{n-m} & , m \leq x \leq n
\end{array} .\right.
$$

In addition to the triangular fuzzy number, some other forms are also used: e.g. L-function, $\Gamma$-function, trapezoidal form or $\Pi$ - function, and others. [13] The value of variable $x$, for which $\mu_{A}(x)=1$ holds, is called the center of fuzzy set $A$. If there are more such values (as in the case of a trapezoidal fuzzy set $A=\operatorname{trapm} f\left[a_{1}, a_{2}, a_{3}, a_{4}\right]$ (Figure 1)) then the set of all values of $x$, for which $\mu_{A}(x)=1$, is called the nucleus of fuzzy set $A$-and it is denoted by

$$
\operatorname{Ker}(A)=\left\{x \mid x \in U \wedge \mu_{A}(x)=1\right\}
$$

The supplier of fuzzy set $A$ is the set of all values of $x$ for which $\mu_{A}(x)>0$, and it is denoted by $\operatorname{supp}(A)=\{x \mid x \in$ $\left.U \wedge \mu_{A}(x)>0\right\}$.

For the practical application of the fuzzy logic in managing systems, of special importance is the existence of a fuzzy set along with one element, for which the value of the function of affiliation is equal to 1. [4,7],

A given fuzzy set $A$, with $\alpha \in[0,1], A_{\alpha}=\{x \in$ $\left.X \mid \mu_{A}(x) \geq \alpha\right\}$ is called the $\alpha$-section of fuzzy set $A$, Figure (1). By using the $\alpha$ section, function of affiliation $\mu_{A}(x)$ of fuzzy set $A$ can be broken down into an infinite number of functions of affiliation of the rectangular form $\alpha \wedge \chi_{A_{\alpha}}(x)$, where $\chi_{A_{\alpha}}(x)$ is a characteristic function of set $A_{\alpha}$.

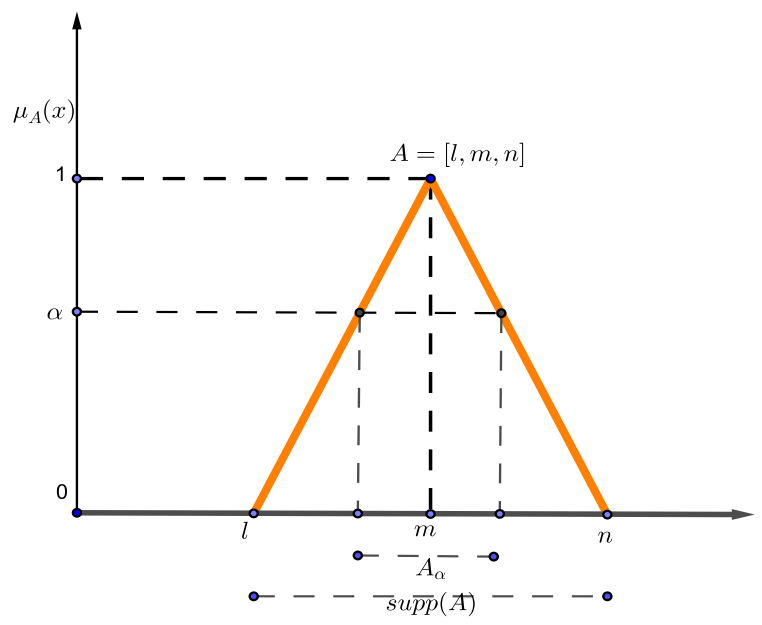

Figure 1. Triangular and trapezoidal forms of a function of affiliation to a fuzzy number.

A structure $P: x$ is $A$, where $x \in U$ is a linguistic variable, $A \in \mathcal{A}$ is the space of linguistic values that variable $x$ can take, that is, the space of fuzzy sets defined over the domain $U$, is called a fuzzy proposition. If we put two fuzzy propositions in a mutual relationship, then a fuzzy relation is created. The fuzzy relation of two propositions, for example,

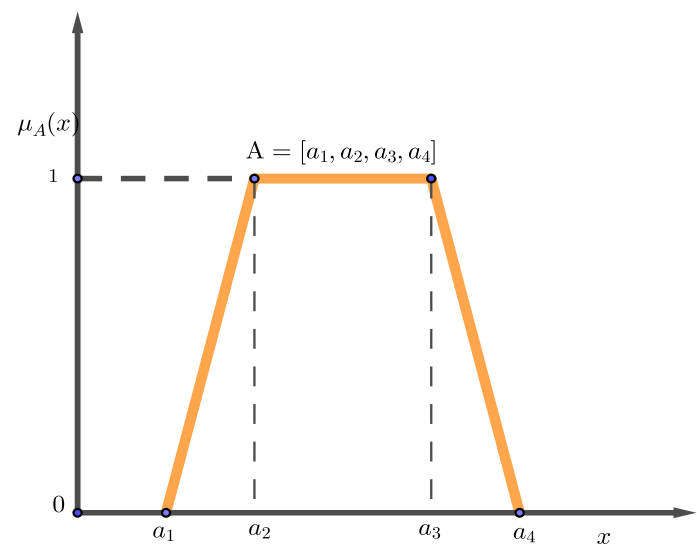

Definition 1 For fuzzy set A over a set of real numbers it is said that it is a fuzzy number if:

1. $A$ is a convex set,

2. There is exactly one $x \in \mathbb{R}$ such that $\mu_{A}(x)=1$, $\operatorname{Ker}(A)=x$,

3. Function of affiliation $\mu_{A}(x)$ for $x \in \mathbb{R}$ is continuous at least in parts [8].

It follows from the definition that a trapezoidal fuzzy set is not a fuzzy number, but a segment, although this is often neglected for practical reasons. Zadeh's principle of enlargement is also important here. [8].

Definition 2. Let $A_{1}, A_{2}, \ldots, A_{n}$ be fuzzy subsets of classical sets $X_{1}, X_{2}, \ldots, X_{n}$ respectively, and let function $\mathrm{f}$ be given as: $f: X_{1} \times X_{2} \times \ldots \times X_{n} \rightarrow Y$ such that for every nnumber $\left(x_{1}, x_{2}, \ldots, x_{n}\right) \in X_{1} \times X_{2} \times \ldots \times X_{n}$ the following applies: $f\left(x_{1}, x_{2}, \ldots, x_{n}\right)=y \in Y$.

Then $B=f\left(A_{1}, A_{2}, \ldots, A_{n}\right)$ is a fuzzy subset of $Y$ whose function of affiliation is the following:

$$
\mu_{B}= \begin{cases}\sup _{y} \min \left\{\mu_{A_{1}}\left(x_{1}\right), \ldots, \mu_{A_{n}}\left(x_{n}\right)\right\}, & \text { if there is } y=f\left(x_{1}, \ldots, x_{n}\right) \\ 0, & \text { otherwise }\end{cases}
$$

If $A$ and $B$ are fuzzy subsets of universal sets $U_{1}$ and $U_{2}$, then the Cartesian product of $A \times B$ is a fuzzy reation $R$ on set $U_{1} \times U_{2}$ and we denote it as $R=A \times B$, and for function $\mu_{R}: U_{1} \times U_{2} \rightarrow[0,1]$ the following is valid:

$$
\mu_{R}(x)=\min \left[\mu_{A}(x), \mu_{B}(y)\right]
$$

In general, if $A_{1}, A_{2}, \ldots, A_{n}$ are fuzzy subsets of universal sets $U_{1}, U_{2}, \ldots, U_{n}$, respectively, then their Cartesian product $A_{1} \times A_{2} \times \ldots \times A_{n}$, is the fuzzy relation on set $U_{1} \times U_{2} \times \ldots \times$ $U_{n}$. Let us denote it by $R=A_{1} \times A_{2} \times \ldots \times A_{n}$ and, [4] applies:

$$
\mu_{R}\left(x_{1}, x_{2}, \ldots, x_{n}\right)=\min \left\{\mu_{A_{i}}\left(x_{i}\right) \mid x_{i} \in U_{i}, i=1,2, \ldots\right.
$$

has the following form:

$$
R: x \text { is } A \text { and } y \text { is } B,
$$

where $x \in U_{1}, y \in U_{2}$ are linguistic variables, and $A \in$ $\mathcal{A}, B \in \mathcal{B}$ are fuzzy sets. In a fuzzy relation there appears 
function $\mu_{R}(x, y):[0,1] \times[0,1] \rightarrow[0,1]$ which represents the "truth" of relation $R$ :

$$
\begin{gathered}
R=\left\{\left(\mu_{R}(x, y), x, y\right) \mid x \in U_{1}, y \in U_{2}\right\}, \\
\mu_{R}(x)=\rho\left\{\mu_{A}(x), \mu_{B}(y)\right\}
\end{gathered}
$$

where $\rho\{$.$\} represents one of the operators which forms$ function $\mu_{R}(x, y)$ and which then directly affects the structure of the fuzzy controller [14,15].

The fuzzy rule base is the central part of the fuzzy controller. The fuzzy rule as the main part of the base has the following form: If $R$ then $P$, where $R$ and $P$ represent the fuzzy relation or fuzzy proposition. The fuzzy rule needs to be given meaning in terms of the truth of proposition $P$, after determining the affiliation function $\mu_{R}($.$) of relation R$. In fuzzy controllers, there are usually fuzzy implications of type min and product which give the meaning $\mu_{R \rightarrow P}=$ $\min \left(\mu_{R}, \mu_{P}\right), \mu_{R \rightarrow P}=\mu_{R} * \mu_{P}$, to the rule "if $\mathrm{R}$, then $\mathrm{P} "$ where $R \rightarrow P$ is a rule. The min operation of the fuzzy implication is also called the Mamdani implication and it is one of the most commonly used implications in a fuzzy controler. $[14,15]$

\section{A Fuzzy Process Modelling}

A theory based on fuzzy sets allows for the reasoning with non-specific information. The original idea of making decisions based on imprecise data by combining linguistic rules comes from Zade [5] who calls these reasoning approximate or fuzzy reasoning.

Fuzzy reasoning is a procedure of inference based on premises (lemmas) that contain linguistic expressions. The central feature of this theory is a representation of propositions as commands that join the fuzzy sets. The input and output variables in fuzzy systems are linguistic variables. We write a linguistic variable in the form of $(x, F, U, M)[7]$, where:

1. $\mathrm{x}$ is the name of the variable,

2. $F$ is the space of linguistic values that variable $x$ can take, that is, the space of the fuzzy sets over the domain $\mathrm{U}$,

3. $\mathrm{U}$ is the quantitative domain of consideration of variable $\mathrm{x}$ over which it assumes linguistic values,

4. $\mathrm{M}$ is a semantic function (rule) of connecting space

F with domain $U$ i.e. $M: U \rightarrow F$ [7]. All possible values of the input and output variables are associated with the appropriate degree of affiliation [6].

A FLC (Fuzzy Logic Control) technique of application of fuzzy sets and fuzzy logic has a significant role in solving various problems in the theory of decision making. It is mainly based on if-then rules that describe complex and insufficiently precise problems with the help of fuzzy sets.

Each FLC process has input and output values that are given in the form of linguistic variables.

Let linguistic variable $x$ be given by $(x, \mathcal{A}(x), \mathcal{U}, \mathcal{M})$, and classified in the domain of definition $\mathcal{U}=[0, a], a \in \mathbb{R}$ by a family of $p$ fuzzy numbers

$$
\mathcal{A}(x)=\left\{\left(x, \mu_{A_{i}}(x)\right) \mid x \in A_{i}, A_{i} \subset \mathcal{U} ; i=1,2,3, \ldots, p\right\}
$$

and affiliation functions $\mu_{A_{i}}(x)$, written in a triangular, trapezoidal or $\Gamma$ form. Let us mark with $B_{i}, i=1,2, \ldots, p-1$ the points of intersection of fuzzy numbers $A_{i}, A_{i+1}, i=$ $1,2, \ldots, p-1$, and with $a_{1}, a_{2}, ., a_{i-1}, a_{i}, a_{i+1}, \ldots, a_{p-1}$ their projections on the $O x$ axis. Points $a_{i}$ divide segment $U=[0, a]$ into disjoint subintervals.

Definition 1. Let fuzzy number $A_{i} \in \mathcal{A}(x)$ be defined in domain $U_{i} \subseteq U$. Interval $\left[a_{i-1}, a_{i}\right]=\left\{x \mid a_{i-1} \leq x \leq a_{i}, x \in\right.$ $\left.A_{i}\right\} \subseteq U_{i}$ is the interval of the dominant affiliation of variable $x$ to fuzzy number $A_{i}$ in relation to fuzzy numbers $A_{i-1}, A_{i+1} \in \mathcal{A}(x)$ defined (or partially defined) in domain $U_{i}$, if for all values of variable $x \in\left[a_{i-1}, a_{i}\right]$ the following is valid:

$$
\mu_{A_{i}}(x)=\max _{x \in\left[a_{i-1}, a_{i}\right]}\left\{\mu_{A_{i-1}}(x), \mu_{A_{i}}(x), \mu_{A_{i+1}}(x)\right\} .
$$

Points $x=a_{i}$ are the points of change of the dominant affiliation in the domain $U_{i}$ of fuzzy numbers $A_{i-1}, A_{i}, A_{i+1}$ (Figure 2) [11].

To show that $\left[a_{1}, a_{2}\right] \subseteq U_{i}$ is the interval of dominant affiliation of variable $x$ to fuzzy number $A$ as defined in domain $U_{A}$ we write in short the following: $\operatorname{Da} f_{x}(A)$. Therefore,

$$
\begin{gathered}
\operatorname{Daf}_{x}(A)=\left[a_{1}, a_{2}\right] \\
=\left\{x \mid a_{1} \leq x \leq a_{2}, x \in A\right\} \subset U_{A} .
\end{gathered}
$$

If it is known which variable the interval of dominant affiliation refers to, we can omit it in the record, so instead of $\operatorname{Daf}_{x}(A)$ we can write down $\operatorname{Daf}(A)$.

Definition 2. If fuzzy set $A \in \mathcal{A}(x)$ is defined in domain $U_{A}$ of the universal set, then the difference $U_{A} \backslash D a f_{x}(A)=$ $\overline{D a f_{x}(A)}$ is called the complement of affiliation of dominant set $D a f_{x}(A)$ in universal domain $U_{A}$ of variable $\mathrm{x}$, and we define it as follows

$$
\overline{D a f_{x}(A)}=U_{A} \backslash D_{x}(A)=\left\{x \mid x \in A \wedge x \notin D a f_{x}(A)\right\}
$$

\section{Mathematical Logical Argumentation for Clustering Possible States of the Output Variable}

Let the value of variable $x$ be evaluated by family $\mathcal{A}(x)=\left\{A_{1}, A_{2}, \ldots, A_{p}\right\}$ of $p$ fuzzy numbers, and variable $y$ by family $\mathcal{B}(y)=\left\{B_{1}, B_{2}, \ldots, B_{q}\right\}$ of $q$ fuzzy numbers. A Cartesian product

$$
\mathcal{A}(x) \times \mathcal{B}(y)=\left\{\left(A_{i}, B_{j}\right) \mid A_{i} \in \mathcal{A}(x), B_{j} \in \mathcal{B}(y)\right\},
$$

where $i=1,2, \ldots, p ; j=1,2, \ldots, q$ determins the set of all pairs of fuzzy numbers for which the values of variables $x$ and $y$ are evaluated. Each pair of fuzzy numbers represents one state of evaluation of the output variable $u$ in the fuzzy model and generates one "if... then" inference rule. Let the value of the output variable $u$ of the model be evaluated by 
family $\mathcal{U}(u)$ of $r$ fuzzy attribute.

In order to reduce the number of rules on the basis of which we make inferences about states that describe the possible classifications of values of the output variable in the model, it is necessary to group descriptive states into clusters (classes). Let us consider a method of grouping the output states in which the value of an output variable has been described.

The method is based on grouping states based on a unique value that can be associated with each state that describes the output variable. This value is called a characteristic value of the state, and it is denoted as $\chi\left(s_{i}\right) \in \mathcal{K}$ which assigns a real number from set $K \subseteq \mathbb{R}$ to each state $s$ from the set of all states.

\subsection{Characteristic Elements of a Fuzzy Number on the Dominant Affiliation Interval}

Let the value of input variable $x$ given by $(x, \mathcal{A}(x), \mathcal{U}, \mathcal{M})$ be classified in relation to the average value, by the family of fuzzy attributes

$$
\begin{gathered}
\mathcal{A}(x)=\{\text { less than average, average value, } \\
\text { greater than average }\}=\left\{A_{1}, A_{2}, A_{3}\right\} .
\end{gathered}
$$

Let evaluation $A_{1}=$ less than average correspond to unsustainable, $A_{2}=$ average value correspond to tolerable, and $A_{3}=$ greater than average correspond to sustainable state and let

$$
\begin{gathered}
\operatorname{Daf}_{x}\left(A_{1}\right)=\left[0, a_{1}\right), \operatorname{Daf}_{x}\left(A_{2}\right)=\left[a_{1}, a_{2}\right], \\
\operatorname{Daf}_{x}\left(A_{3}\right)=\left(a_{2}, a\right]
\end{gathered}
$$

be intervals of dominant affiliation of variable $x$ for fuzzy numbers, $A_{1}, A_{2}, A_{3}$ respectively, (Figure 5), so that order $0<a_{1}<a_{2}<1$ is valid. [11]

Let us now define function $\chi_{x}: \mathcal{A}(x) \rightarrow K \subseteq \mathbb{R}$ which associates one real value $k$ from set $K \subseteq \mathbb{R}$ to each fuzzy number $A_{i} \in \mathcal{A}(x)$ on the interval of dominant affiliation of the variable $x$, so that

1. Set $K=\{-1,0,1\}$, if the value of domain $\mathcal{U}$ of input variable $x$ is classified by a family of three fuzzy attributes, according to the rule.

$$
\chi_{x}\left(A_{i}\right)= \begin{cases}1, & \text { if } A_{i} \text { describes sustainable state } \forall x \in \operatorname{Daf}\left(A_{i}\right) \\ 0, & \text { if } A_{i} \text { describes tolerable state } \forall x \in \operatorname{Daf}\left(A_{i}\right) \\ -1, & \text { if } A_{i} \text { describes unsustainable state } \forall x \in \operatorname{Daf}\left(A_{i}\right)\end{cases}
$$

2. Set $K=\{-2,-1,0,1,2\}$, if the value of domain $\mathcal{U}$ of output variable $x$ is classified by family $\mathcal{A}(x)$ of five fuzzy attributes

$$
\mathcal{A}(x)\left\{\left(x, \mu_{A_{i}}(x)\right) \mid x \in A_{i}, A_{i} \subseteq U, i=1,2, \ldots, 5\right\}=\left\{A_{1}, A_{2}, A_{3}, A_{4}, A_{5}\right\}=\left\{A_{1} \equiv \text { alarming state, } A_{2} \equiv\right.
$$

intolerable state, $A_{3} \equiv$ tolerable state, $A_{4} \equiv \operatorname{good}$ state, $A_{5} \equiv$ sustainable state $\}$ according to the rule

$$
\chi_{x}\left(A_{i}\right)=\left\{\begin{array}{llll}
-2 & \text {, if } A_{i} & \text { describes alarming state } & \forall x \in D a f_{A_{1}} \subset U_{1} \\
-1 & \text {, if } A_{i} & \text { describes unsustainable state } & \forall x \in D a f_{A_{2}} \subset U_{2} \\
0 & \text {, if } A_{i} & \text { describes tolerable state } & \forall x \in D a f_{A_{3}} \subset U_{3} \\
1 & \text {, if } A_{i} & \text { describes good state } & \forall x \in D a f_{A_{4}} \subset U_{4} \\
2 & \text {, if } A_{i} & \text { describes sustaianble state } & \forall x \in D a f_{A_{5}} \subset U_{5}
\end{array}\right.
$$

where $\operatorname{Daf}_{x}\left(A_{i}\right)=\left[a_{i-1} ; a_{i}\right], i=1,2,3,4,5 a_{0}=0, \wedge a_{5}=a$, and $\cup_{i=1}^{5} D_{i}=U, U=[0, a], a \in \mathbb{R}$ are the intervals of dominant affiliation of corresponding fuzzy number $A_{i}$ defined over domain $U_{i}$.

3 . In case the value of input variable $x$ is classified by family $\mathcal{A}(x)$ of $p$ fuzzy numbers (atributes) and let $p \equiv$ $1(\bmod 2), p \in \mathbb{N}$ then set $K=\left\{0, \pm 1, \pm 2, \ldots, \pm \frac{p-1}{2}\right\}$, i.e. the following is valid: $\chi_{x}\left(A_{i}\right) \in K=\left\{0, \pm 1, \pm 2, \ldots, \pm \frac{p-1}{2}\right\}$.

Definition 3. Function $\chi_{x}: \mathcal{A}(x) \rightarrow K \subseteq \mathbb{R}$ is called the characteristic function of fuzzy number $A_{i} \in \mathcal{A}(x)$ on the interval of dominant affiliation $\operatorname{Da} f_{x}\left(A_{i}\right)$ of variable $x$, and real number $k \in K$ is called the characteristic value of fuzzy number $A_{i}$ on the interval of dominant affiliation.

Let $A_{1}, A_{2} \in \mathcal{A}(x)$ be two fuzzy numbers from the family by which the values of variable $x$ are classified.

Definition 4. Two fuzzy numbers $A_{1}, A_{2}$ which describe the values of variable $x$, and which have the same characteristic values $\chi\left(A_{1}\right)=\chi\left(A_{2}\right)$ are considered to be equal. Symbolically put:

$$
\forall A_{1}, A_{2} \in \mathcal{A}(x), \chi_{x}\left(A_{1}\right)=\chi_{x}\left(A_{2}\right) \Leftrightarrow A_{1}=A_{2}
$$

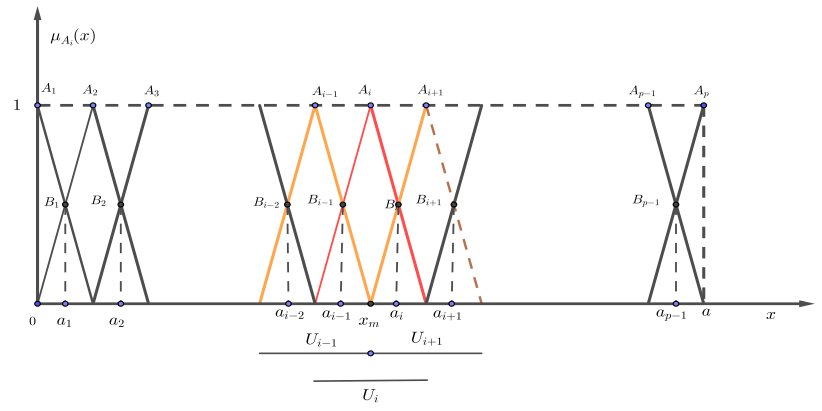

Figure 2. Family of fuzzy numbers $\mathcal{A}(x)$ and intervals of dominant affiliation.

Let us define another characteristic value of variable $x$ evaluated by fuzzy number $A_{i}$ on the dominant affiliation interval, $x \in \operatorname{Daf}\left(A_{i}\right)$. This value is called a weight impact of fuzzy number $A_{i} \in \mathcal{A}(x)$ in the quantification of variable $x$.

Let variable $x$ be given by $(x, \mathcal{A}(x), \mathcal{U}, \mathcal{M})$, and classified in the domain of definition $\mathcal{U}=[0, a], a \in \mathbb{R}$ by family of $p$ fuzzy numbers $\mathcal{A}(x)=\left\{\left(x, \mu_{A_{i}}(x)\right) \mid x \in A_{i}, A_{i} \subset \mathcal{U} ; i=\right.$ $1,2,3, \ldots, p\}$ and affiliation functions $\mu_{A_{i}}(x)$, written in 
triangular, trapezoidal or $\Gamma$ forms. Let $\operatorname{Daf}\left(A_{i}\right)=$ $\left[a_{i-1} ; a_{i}\right], a_{0}=0, a_{p}=a, a \in \mathbb{R}$ be the interval of dominant affiliation of fuzzy numbers $A_{i} \in \mathcal{A}(x)$. For the observed fuzzy number $A_{i}$ let us notice the following values of variable $x$ in the interval of dominant affiliation $\operatorname{Daf}\left(A_{i}\right)=$ $\left[a_{i-1} ; a_{i}\right]$ in the following order:

$$
m_{2}=\frac{a_{i-1}+a_{i}}{2}, m_{1}=\frac{a_{i-1}+m_{2}}{2}, m_{3}=\frac{m_{2}+a_{i}}{2},
$$

and $\lambda=|\mathcal{A}(x)|$ - the number of fuzzy attributes of family $\mathcal{A}(x)$ be which variable $x$ is classified.

Definition 5. The weight impact of fuzzy number $A_{i} \in$ $\mathcal{A}(x)$ in the quantification of variable $x$, on the interval of dominant affiliation $\operatorname{Daf}\left(A_{i}\right)=\left[a_{i-1} ; a_{i}\right]$, - let us denote it by $\rho_{x}\left(A_{i}\right),-$ represents the product of the characteristic value $\chi_{x}$ of fuzzy number $A_{i}$ and the values of the weighted means of the dominant affiliation interval, $\tau_{x}\left(A_{i}\right)$. Symbolically put: $\rho_{x}\left(A_{i}\right)=\chi_{x}\left(A_{i}\right) \cdot \tau_{x}\left(A_{i}\right)$ where

$$
\tau_{x}\left(A_{i}\right)=\frac{1}{\lambda} \cdot \frac{\sum_{j=1}^{3} m_{j} \cdot \mu_{A_{i}}\left(m_{j}\right)}{m_{1}+m_{2}+m_{3}}, \lambda=|\mathcal{A}(x)|
$$

is the value of weighted means, and $\lambda=|\mathcal{A}(x)|$ is the number of fuzzy attributes of family $\mathcal{A}(x)$ by which the value of variable $x$ is classified. If variable $x$ on its universal

$$
M_{x}(\mathcal{A}(x))=\begin{aligned}
& A_{1} \\
& A_{2} \\
& \cdots \\
& A_{p}
\end{aligned}\left[\begin{array}{ll}
\chi_{x}\left(A_{1}\right) & \tau_{x}\left(A_{1}\right) \\
\chi_{x}\left(A_{2}\right) & \tau_{x}\left(A_{2}\right) \\
\chi_{x}\left(A_{p}\right) & \tau_{x}\left(A_{p}\right)
\end{array}\right] \text { or } M_{x}(\mathcal{A}(x))=\left[\begin{array}{l}
\rho_{x}\left(A_{1}\right) \\
\rho_{x}\left(A_{2}\right) \\
\cdots \\
\rho_{x}\left(A_{p}\right)
\end{array}\right]
$$

\subsection{Characteristic Elements of a Pair of Fuzzy Numbers on a Rectangle of Dominant Affiliation}

Let the output variable in a fuzzy model be impacted by two input variables $\left(x, \mathcal{A}(x), \mathcal{U}, \mathcal{M}_{1}\right), \quad\left(y, \mathcal{B}(y), \mathcal{V}, \mathcal{M}_{2}\right)$ defined in domains $\mathcal{U}$ and $\mathcal{V}$ by families of fuzzy

$$
\begin{aligned}
& \mathcal{A}(x)=\left\{\left(x, \mu_{A_{i}}(x)\right) \mid x \in A_{i}, A_{i} \subset U_{i} \subset \mathcal{U}, i=1,2, \ldots, p\right\} . \\
& \mathcal{B}(y)=\left\{\left(y, \mu_{B_{i}}(y)\right) \mid y \in B_{i}, B_{i} \subset V_{j} \subset \mathcal{V}, i=1,2, \ldots, q\right\} .
\end{aligned}
$$

respectively. With a Cartesian product: $\mathcal{A}(x) \times \mathcal{B}(y)=$ $\left\{\left(A_{i}, B_{j}\right) \mid A_{i} \subset \mathcal{U}, B_{j} \subset \mathcal{V}\right\}$ of the families of fuzzy numbers $\mathcal{A}(x), \mathcal{B}(y)$, defined in domains $\mathcal{U}[0, a], \mathcal{V}=[0, b], a, b \in \mathbb{R}$ all pairs $\left(A_{i}, B_{j}\right)$ of fuzzy numbers $A_{i} \in \mathcal{A}(x), B_{j} \in \mathcal{B}(y)$ are determined by which the values of input variables $x$ and $y$ have been determined in the fuzzy model. Let points $0=a_{0}, a_{1}, \ldots, a_{i-1}, a_{i}, \ldots, a_{p}=a$, divide domain $U=[0, a]$ set is classified by fuzzy numbers so that it describes a more favorable state $\AA_{i}$, then its value is ever so smaller, and we replace the mean $m_{j}$ with a reciprocal value, $\frac{1}{m_{j}}$.

In this way, to each fuzzy number $A_{i} \in \mathcal{A}(x)$ there can be assigned its characteristic elements on the dominant affiliation interval that represent it in the evaluation of the output variable of the model, which is called a feature of fuzzy number $A_{i}$ in the evaluation of the model of variable $x$, and that is:

1. $\chi_{x}\left(A_{i}\right)$ - characteristic value of fuzzy number, $\chi_{x}\left(A_{i}\right) \in$ $K \subseteq \mathbb{Z}$

2. $\left(\tau_{x}\left(A_{i}\right)\right)$ - value of weighted means of a fuzzy number on the interval of dominant affiliation.

3. $\left(\rho_{x}\left(A_{i}\right)\right)$ - the value of the weight impact of fuzzy number on the evaluation of the output variable.

The features of fuzzy number $A_{i} \in \mathcal{A}(x)$ can be represented in the form of vector $v\left(A_{i}\right)=\left(\chi_{x}\left(A_{i}\right),\left(\tau_{x}\left(A_{i}\right)\right)\right)^{T}$. If variable $x$ is classified by family $\mathcal{A}(x)=\left\{A_{1}, A_{2}, \ldots, A_{p}\right\}$ of $p$ fuzzy attributes at the intervals of the dominant affiliation of the feature of family $\mathcal{A}(x)$ of fuzzy attribute, then we write it down in the matrix form as follows:

into $p$ disjoint intervals $\left[a_{i-1}, a_{i}\right]$ of the dominant affiliation to fuzzy number $A_{i}, i=1,2, \ldots, p$, and let points $0=$ $b_{0}, b_{1}, \ldots, b_{i-1}, b_{i}, \ldots, b_{q}=b$, divide domain $V=[0, b]$ into $q$ disjoint intervals $\left[b_{j-1}, b_{j}\right]$ of the dominant affiliation to fuzzy number $B_{j}, j=1,2, \ldots, q$.

Then lines $x=a_{i}, y=b_{j}$ divide domain

$P=\{(x, y) \mid x \in[0, a]=\mathcal{U}, y \in[0, b]=\mathcal{V}\}=\mathcal{U} \times \mathcal{V}$

into $p \cdot q$ rectangle $\Delta p_{i j}=\left[a_{i-1}, a_{i}\right] \times\left[b_{j-1}, b_{j}\right] \subseteq U_{i} \times V_{j}$ where $\mathcal{U}_{i}, \mathcal{V}_{j}$ are the definition domains of fuzzy numbers $A_{i}, B_{j}$, respectively.

Definition 6. Let $\operatorname{Daf}_{A_{i}}(x)=\left[a_{i-1}, a_{i}\right], D a f_{B_{j}}(x)=$ $\left[b_{j-1}, b_{j}\right]$ be the intervals of dominant affiliation of fuzzy numbers $A_{i} \subset \mathcal{U}_{i}, B_{j} \subset \mathcal{V}_{j}$. Rectangle

$$
\begin{aligned}
\Delta p_{i j}= & {\left[a_{i-1}, a_{i}\right] \times\left[b_{j-1}, b_{j}\right]=\left\{(x, y) \mid \forall x \in \operatorname{Daf} f_{x}\left(A_{i}\right) \Rightarrow \max \left\{\mu_{A_{1}}(x), \ldots, \mu_{A_{p}}(x)\right.\right.} \\
& \left.\left.=\mu_{A_{i}}(x)\right\} \wedge \forall y \in \operatorname{Daf}\left(B_{j}\right) \Rightarrow \max \left\{\mu_{B_{1}}(y), \ldots, \mu_{B_{q}}(y)\right\}=\mu_{B_{j}}(y)\right\},
\end{aligned}
$$

is called the rectangle of dominant affiliation of a pair of fuzzy numbers $\left(A_{i}, B_{j}\right)$ and it is denoted by $D a f_{x, y}\left(A_{i}, B_{j}\right)$. If it is known which variables it refers to, then we write $\operatorname{Daf}\left(A_{i}, B_{j}\right)$ (Figure 3 ). The weighted mean of the pair of fuzzy numbers $\left(A_{i}, B_{j}\right)$ on the interval of the dominant rectangle is defined as follows. 


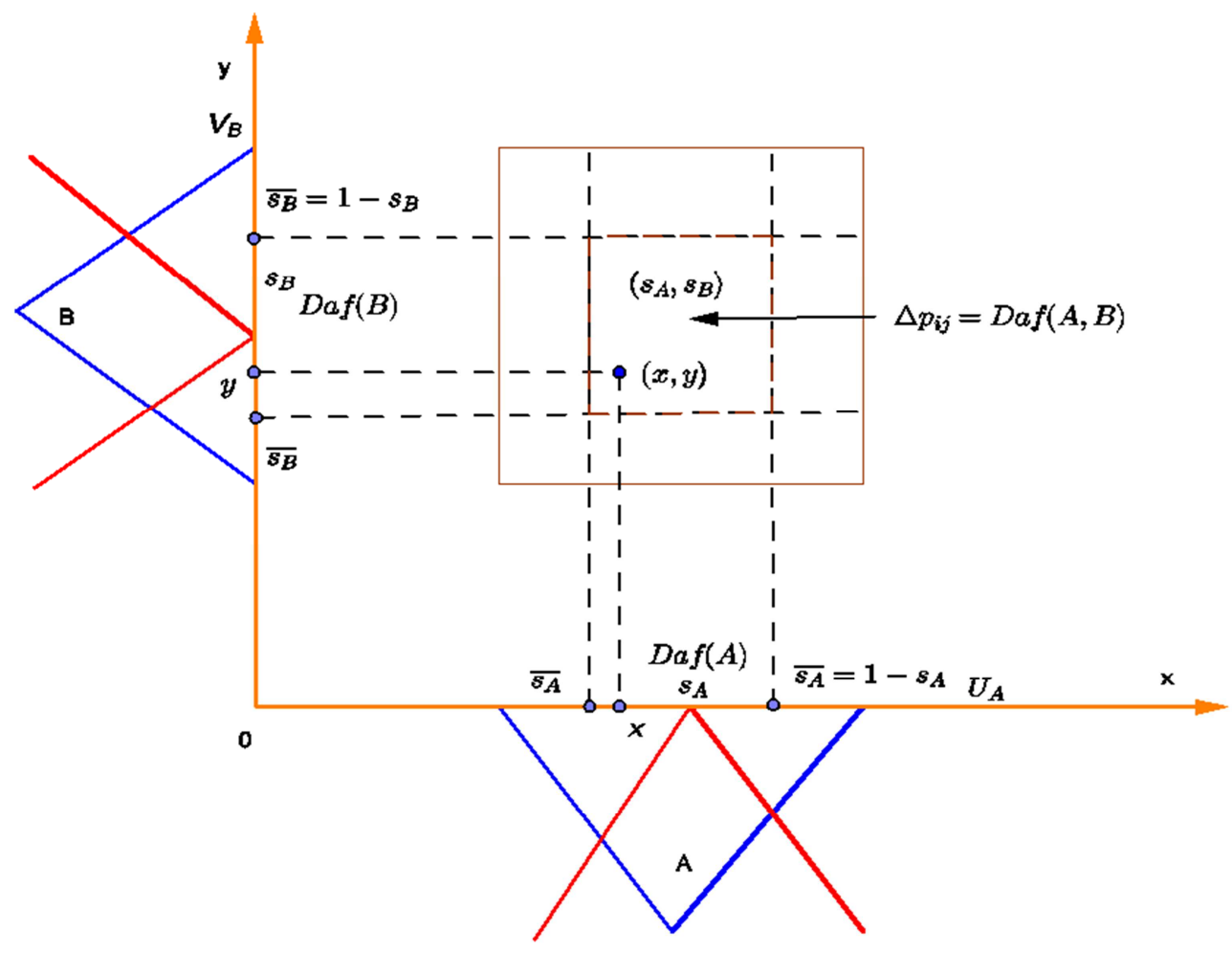

Figure 3. Interval of dominant affiliation of a pair of fuzzy numbers.

Definition 7. The weighted mean of pair $\left(A_{i}, B_{j}\right) \in$ $\mathcal{A}(x) \times \mathcal{B}(y)$ of fuzzy numbers on the rectangle of dominant affiliation $\Delta p_{i j}=\left[a_{i-1}, a_{i}\right] \times\left[b_{j-1}, b_{j}\right]$ is defined by a relation

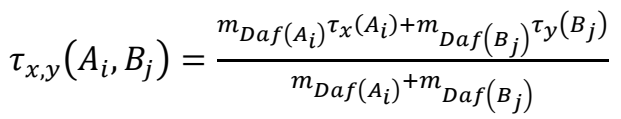

where $m_{i}=\frac{a_{i-1}+a_{i}}{2}$ is the mean of the interval of dominant affiliation of fuzzy number $A_{i}$, i.e. of fuzzy number $B_{j}$, and $\tau_{x}\left(A_{i}\right)$ is the weighted value of the mean of fuzzy number $A_{i}$ i.e. of $B_{j}$ on the interval of dominant affiliation.

If fuzzy number $A_{i}$ and (or $B_{j}$ ) belongs to a family that is classified on its universal set $U$ in a such way that it describes the more favorable state, and that is when the value of the variable is smaller, then we replace the mean of interval $m_{i}$ of the dominant affiliation with its reciprocal value.

Let us also define the function of the weight impact of the pair $\left(A_{i}, B_{j}\right)$ of fuzzy numbers $A_{i}, B_{i}$ on the description of the state of the output variable of the fuzzy model.

Definition 8. Function $\rho: \mathcal{A}(x) \times \mathcal{B}(y) \rightarrow \mathbb{R}$ which for each pair of fuzzy numbers $\left(A_{i}, B_{j}\right) \in \mathcal{A}(x) \times \mathcal{B}(y)$ by which variables $x \in \operatorname{Daf}\left(A_{i}\right)=\left[a_{1}, a_{2}\right]$ and $y \in \operatorname{Daf}\left(B_{j}\right)=$ $\left[b_{1}, b_{2}\right]$ are evalated - associates real number $k$ according to the following formula

$$
\begin{aligned}
\rho_{x, y}\left(A_{i}, B_{j}\right) & =\rho_{x}\left(A_{i}\right)+\rho_{y}\left(B_{j}\right) \\
& =\chi_{x}\left(A_{i}\right) \tau_{x}\left(A_{i}\right)+\chi_{y}\left(B_{j}\right) \tau_{y}\left(B_{j}\right)
\end{aligned}
$$

is called the function of the weight impact of a pair of fuzzy numbers $\left(A_{i}, B_{j}\right)$ on the interval of dominant affiliation $(x, y) \in \operatorname{Daf}\left(A_{i}, B_{j}\right)$,

Based on the calculated value of the weight impact function and the value of the weighted means, the characteristic function of the pair of fuzzy numbers $\left(A_{i}, B_{j}\right)$ on the rectangle of dominant affiliation $\operatorname{Da} f_{x, y}\left(A_{i}, B_{j}\right)$ is defined as follows:

Definition 9. Function $\chi_{x, y}\left(A_{i}, B_{j}\right): \mathcal{A}(x) \times \mathcal{B}(y) \rightarrow K$ defined by relation

$$
\chi_{x, y}\left(A_{i}, B_{j}\right)=\frac{\chi_{x}\left(A_{i}\right) \tau_{x}\left(A_{i}\right)+\chi_{y}\left(B_{j}\right) \tau_{y}\left(B_{j}\right)}{\tau_{x, y}\left(A_{i}, B_{j}\right)}=\frac{\rho_{x, y}\left(A_{i}, B_{j}\right)}{\tau_{x, y}\left(A_{i}, B_{j}\right)}
$$

is called the characteristic function of the pair of fuzzy numbers $\left(A_{i}, B_{j}\right)$ on the interval of dominant affiliation, $\operatorname{Daf}\left(A_{i}, B_{j}\right)$, and real number $k \in K$ is the characteristic value of the pair of fuzzy numbers.

The characteristic elements of the pairs of fuzzy numbers $\left(A_{i}, B_{j}\right)$ of the product of $\mathcal{A}_{i} \times \mathcal{B}_{j}$ of families $\mathcal{A}(x), \mathcal{B}(y)$ is written in the matrix form:

$$
\begin{aligned}
\tau_{x, y}\left(\mathcal{A}_{i} \times \mathcal{B}_{j}\right) & =\left[\begin{array}{llll}
\tau_{x}\left(A_{1}, B_{1}\right) & \tau_{x}\left(A_{1}, B_{2}\right) & \ldots & \tau_{x}\left(A_{1}, B_{q}\right) \\
\tau_{x}\left(A_{2}, B_{1}\right) & \tau_{x}\left(A_{2}, B_{2}\right) & \ldots & \tau_{x}\left(A_{2}, B_{q}\right) \\
\ldots & \ldots & \ldots & \ldots \\
\tau_{x}\left(A_{p}, B_{1}\right) & \tau_{x}\left(A_{p}, B_{2}\right) & \ldots & \tau_{x}\left(A_{p}, B_{q}\right)
\end{array}\right], \\
\rho_{x, y}\left(\mathcal{A}_{i} \times \mathcal{B}_{j}\right) & =\left[\begin{array}{llll}
\rho_{x}\left(A_{1}, B_{1}\right) & \rho_{x}\left(A_{1}, B_{2}\right) & \ldots & \rho_{x}\left(A_{1}, B_{q}\right) \\
\rho_{x}\left(A_{2}, B_{1}\right) & \rho_{x}\left(A_{2}, B_{2}\right) & \ldots & \rho_{x}\left(A_{2}, B_{q}\right) \\
\ldots & \ldots & \ldots & \ldots \\
\rho_{x}\left(A_{p}, B_{1}\right) & \rho_{x}\left(A_{p}, B_{2}\right) & \ldots & \rho_{x}\left(A_{p}, B_{q}\right)
\end{array}\right]
\end{aligned}
$$


$\chi_{x, y}\left(\mathcal{A}_{i} \times \mathcal{B}_{j}\right)=\left[\begin{array}{llll}\chi_{x}\left(A_{1}, B_{1}\right) & \chi_{x}\left(A_{1}, B_{2}\right) & \cdots & \chi_{x}\left(A_{1}, B_{q}\right) \\ \chi_{x}\left(A_{2}, B_{1}\right) & \chi_{x}\left(A_{2}, B_{2}\right) & \cdots & \chi_{x}\left(A_{2}, B_{q}\right) \\ \cdots & \ldots & \ldots & \ldots \\ \chi_{x}\left(A_{p}, B_{1}\right) & \chi_{x}\left(A_{p}, B_{2}\right) & \cdot & \chi_{x}\left(A_{p}, B_{q}\right)\end{array}\right]$

Theorem 1. Let there be that for the given fuzzy numbers $A_{i}, B_{j}$ the characteristic elements on the intervals of dominant affiliation are $\operatorname{Daf}_{x}\left(A_{i}\right)=\left[a_{i-1}, a_{i}\right]$ and $\operatorname{Da} f_{x}\left(A_{i}\right)=\left[a_{i-1}, a_{i}\right]$, the means of which are $m_{1}=$ $m_{\operatorname{Daf}\left(A_{i}\right)}, m_{2}=m_{\operatorname{Daf}\left(B_{j}\right)}$, respectively; $\chi_{x}\left(A_{i}\right), \tau_{x}\left(A_{i}\right)$ and $\chi_{y}\left(B_{j}\right), \tau_{y}\left(B_{j}\right)$. If $\frac{\tau_{x}\left(A_{i}\right)}{\tau_{y}\left(B_{j}\right)}=l$ is the ratio of the weighted means of fuzzy numbers $A_{i}, B_{j}$ then for the value of characteristic function $\chi_{x, y}\left(A_{i}, B_{j}\right)$ the following evaluation is valid;

$$
\begin{aligned}
& \text { If } \tau_{x}\left(A_{i}\right)=\tau_{y}\left(B_{j}\right) \text { then } \\
& \qquad \begin{array}{r}
\text { If } \tau_{x}\left(A_{i}\right)< \\
\tau_{y}\left(B_{j}\right) \text { then } \\
\left.\chi_{x, y}\left(A_{i}, B_{j}\right)>l \cdot B_{j}\right)=\chi_{x}\left(A_{i}\right)+\chi_{y}\left(B_{j}\right)
\end{array}
\end{aligned}
$$

If

$$
\begin{aligned}
& \tau_{x}\left(A_{i}\right)>\tau_{y}\left(B_{j}\right) \text { then. } \\
& \qquad \chi_{x, y}\left(A_{i}, B_{j}\right)<l \cdot \chi_{x}\left(A_{i}\right)+\chi_{y}\left(B_{j}\right)
\end{aligned}
$$

Proof: From the definition of the characteristic value of the function of a pair of fuzzy numbers $\left(A_{i}, B_{j}\right)$ we have the following:

$$
\begin{gathered}
\chi_{x, y}\left(A_{i}, B_{j}\right)=\frac{\rho_{x, y}\left(A_{i}, B_{j}\right)}{\tau_{x, y}\left(A_{i}, B_{j}\right)}=\frac{\chi_{x}\left(A_{i}\right) \tau_{x}\left(A_{i}\right)+\chi_{y}\left(B_{j}\right) \tau_{y}\left(B_{j}\right)}{\frac{m_{\text {Daf }\left(A_{i}\right)} \tau_{x}\left(A_{i}\right)+m_{\text {Daf }\left(B_{j}\right)} \tau_{y}\left(B_{j}\right)}{m_{\text {Daf }\left(A_{i}\right)}+m_{\text {Daf }\left(B_{j}\right)}}} \\
=\left(m_{\text {Daf }\left(A_{i}\right)}+m_{\text {Daf }\left(B_{j}\right)}\right) \frac{\chi_{x}\left(A_{i}\right) \tau_{x}\left(A_{i}\right)+\chi_{y}\left(B_{j}\right) \tau_{y}\left(B_{j}\right)}{m_{\text {Daf }\left(A_{i}\right)} \tau_{x}\left(A_{i}\right)+m_{\text {Daf }\left(B_{j}\right)} \tau_{y}\left(B_{j}\right)} \\
=\left(m_{1}+m_{2}\right)\left(\frac{\chi_{x}\left(A_{i}\right) \tau_{x}\left(A_{i}\right)}{m_{1} \tau_{x}\left(A_{i}\right)+m_{2} \tau_{y}\left(B_{j}\right)}+\frac{\chi_{y}\left(B_{i j}\right) \tau_{y}\left(B_{j}\right)}{m_{1} \tau_{x}\left(A_{i}\right)+m_{2} \tau_{y}\left(B_{j}\right)}\right) \\
=\left(m_{1}+m_{2}\right)\left(\frac{\chi_{x}\left(A_{i}\right)}{m_{1}+m_{2} \frac{\tau_{y}\left(B_{j}\right)}{\tau_{x}\left(A_{i}\right)}}\right. \\
\left.+\frac{\chi_{y}\left(B_{j}\right)}{m_{1} \frac{\tau_{x}\left(A_{i}\right)}{\tau_{y}\left(B_{j}\right)}+m_{2}}\right)
\end{gathered}
$$

Let us assume that $\frac{\tau_{x}\left(A_{i}\right)}{\tau_{y}\left(B_{j}\right)}=l, l \in \mathcal{R}$, then it follows that $\frac{1}{l}=\frac{\tau_{y}\left(B_{j}\right)}{\tau_{x}\left(A_{i}\right)}$. The last equation transforms into a form which is easier to follow:

$$
\begin{aligned}
\chi_{x, y}\left(A_{i}, B_{j}\right) & =\left(m_{1}+m_{2}\right)\left(\frac{\chi_{x}\left(A_{i}\right)}{m_{1}+\frac{1}{l} \cdot m_{2}}+\frac{\chi_{y}\left(B_{j}\right)}{m_{1} \cdot l+m_{2}}\right) \\
& =\frac{m_{1}+m_{2}}{m_{1} \cdot l+m_{2}}\left(l \cdot \chi_{x}\left(A_{i}\right)+\chi_{y}\left(B_{j}\right)\right)
\end{aligned}
$$

Depending on the size of the $l \in \mathbb{R}$ ratio, the following cases can be observed:

1. If $\tau_{x}\left(A_{i}\right)=\tau_{y}\left(B_{j}\right)$, i.e. $l=1$ for the characteristic value of the pair $\left(A_{i}, B_{j}\right)$ of fuzzy numbers, the following is holds

$$
\begin{aligned}
\chi_{x, y}\left(A_{i}, B_{j}\right) & =\left(m_{1}+m_{2}\right)\left(\frac{\chi_{x}\left(A_{i}\right)}{m_{1}+m_{2}}+\frac{\chi_{y}\left(B_{j}\right)}{m_{1}+m_{2}}\right) \\
& =\chi_{x}\left(A_{i}\right)+\chi_{y}\left(B_{j}\right)
\end{aligned}
$$

2. If $0<\tau_{x}\left(A_{i}\right)<\tau_{y}\left(B_{j}\right)$, i.e. $0<l<1$, then a series of inequalities $0<l<1 \Leftrightarrow 0<m_{1} \cdot l<m_{1} \Leftrightarrow m_{2}<m_{2}+$ $m_{1} \cdot l<m_{1}+m_{2}$ is followed by inequality $\left(\frac{m_{1}+m_{2}}{m_{1} \cdot l+m_{2}}>1 \wedge\right.$ $m_{1}, m_{2}>0$ ). If we assume that $\frac{m_{1}+m_{2}}{m_{1} \cdot l+m_{2}}=1+h_{1}, h_{1}>0 \in$ $\mathbb{R}^{+}$, then the latter equality can be written in the following form

$$
\begin{gathered}
\chi_{x, y}\left(A_{i}, B_{j}\right)=\left(1+h_{1}\right)\left(l \cdot \chi_{x}\left(A_{i}\right)+\chi_{y}\left(B_{j}\right)\right) \\
=l \cdot \chi_{x}\left(A_{i}\right)+\chi_{y}\left(B_{j}\right)+h_{1}\left(\cdot \chi_{x}\left(A_{i}\right)+\chi_{y}\left(B_{j}\right)\right) \\
>l \cdot \chi_{x}\left(A_{i}\right)+\chi_{y}\left(B_{j}\right)
\end{gathered}
$$

3. If $\tau_{x}\left(A_{i}\right)>\tau_{y}\left(B_{j}\right)$, i.e. $l>1$ then a series of inequalities

$$
l>1 \Leftrightarrow m_{1} \cdot l>m_{1} \Leftrightarrow m_{1} l+m_{2}>m_{1}+m_{2}
$$

is followed by inequality $\frac{m_{1}+m_{2}}{m_{1} \cdot l+m_{2}}<1$ for $m_{1}, m_{2}>0$. If we assume that $\frac{m_{1}+m_{2}}{m_{1} \cdot l+m_{2}}=1-h_{2}, h_{2}>0 \in \mathbb{R}^{+}$, then for the characteristic value of the pair of fuzzy numbers the following evaluation is valid

$$
\begin{aligned}
& \chi_{x, y}\left(A_{i}, B_{j}\right)=\left(1-h_{2}\right)\left(l \cdot \chi_{x}\left(A_{i}\right)+\chi_{y}\left(B_{j}\right)\right) \\
&=l \cdot \chi_{x}\left(A_{i}\right)+\chi_{y}\left(B_{j}\right)-h_{1}\left(\cdot \chi_{x}\left(A_{i}\right)+\chi_{y}\left(B_{j}\right)\right) \\
&<l \cdot \chi_{x}\left(A_{i}\right)+\chi_{y}\left(B_{j}\right) .
\end{aligned}
$$

Let variable $x$ be classified by family $\mathcal{A}(x)$ of $p$ fuzzy numbers, and variable $y$ by family $\mathcal{B}(y)$ of $q$ fuzzy numbers with values of characteristic functions,

$$
\begin{gathered}
\chi_{x}\left(A_{i}\right) \in\left\{ \pm 0, \pm \pm 1, \ldots, \pm\left(\frac{p-1}{2}\right)\right. \\
\chi_{y}\left(B_{j}\right) \in\left\{ \pm 0, \pm \pm 1, \ldots, \pm\left(\frac{q-1}{2}\right)\right\}
\end{gathered}
$$

and let the product of

$$
\begin{gathered}
\mathcal{A}(x) \times \mathcal{B}(y)=\left\{s \mid s_{i, j}=\left(A_{i}, B_{j}\right), A_{i} \in \mathcal{A}(x), B_{j} \in \mathcal{B}(y),\right. \\
i=1,2, \ldots, p ; j=1,2, \ldots, q\}
\end{gathered}
$$

determine all the states of the evaluation of the output variables marked with $x, y$. For each state $s_{i, j}=\left(A_{i}, B_{j}\right)$ there is one corresponding characteristic value, $\chi_{x, y}\left(A_{i}, B_{j}\right)=\chi_{x, y}\left(s_{i, j}\right)$.

Definition 10. For two descriptive states $s_{1}, s_{2}$ from the set of all states $\mathcal{S}$, it is said that they describe the same value of the output variable, if they have equal values of characteristic functions, and we write it down as follows: $s_{1} \equiv s_{2} \Leftrightarrow \chi s_{1}=$ $\chi_{s_{2}}$.

Definition 11. Let $s_{i, j}=\left(A_{i}, B_{j}\right)$ be the evaluated state of the output variable, and $\chi_{x, y}\left(s_{i, j}\right)$ be its characteristic value. 
Integer $k$ for which the following is valid:

$$
k=\chi_{x, y}\left(s_{i, j}\right)=\chi_{x, y}\left(A_{i}, B_{j}\right)=\chi_{x}\left(A_{i}\right)+\chi_{y}\left(B_{j}\right)
$$

is called the center of a base cluster corresponding to state $s_{i, j}$.

Segment $\left[\frac{2 k-1}{2} ; \frac{2 k+1}{2}\right]_{i, j}^{0}$ is called a base cluster with center in $k$ corresponding to descriptive state $s_{i, j}$ of the output variable and it is denoted by $\left[k_{i, j}\right]^{0}$ (Figure 4 ). In this case, the lower limit of base cluster $\left[k_{i, j}\right]^{0}=\left[\frac{2 k-1}{2} ; \frac{2 k+1}{2}\right]_{i, j}^{0}$ is $L l=\frac{2 k-1}{2}$, and the upper limit is $U l=\frac{2 k+1}{2}$.

Example 1. For example, the limits of a base cluster [2] ${ }^{0}$ with the center in 2 are $L l=\frac{3}{2}, U l=\frac{5}{2} \mathrm{tj} .[2]^{0}=\left[\frac{3}{2}, \frac{5}{2}\right]^{0}$.

Definition 12. Set

$$
\begin{gathered}
K^{0}=\left\{\left[k_{i, j}\right]^{0} \mid k_{i, j}=\chi_{x, y}\left(A_{i}, B_{j}\right)=\chi_{x}\left(A_{i}\right)+\chi_{y}\left(B_{j}\right),\right. \\
i=1,2, \ldots, p ; j=1,2, \ldots, q\} \\
=\left\{[0]^{0},[ \pm 1]^{0},[ \pm 2]^{0}, \ldots,\left[ \pm\left(\frac{p+q}{2}-1\right)\right]^{0}\right\}
\end{gathered}
$$

is called a family of base clusters corresponding to descriptive states $s_{i, j}=\left(A_{i}, B_{j}\right)$ of the output variable generated by the Cartesian product of the families of fuzzy numbers $\mathcal{A}(x), \mathcal{B}(y)$ by which input variables $x, y$ of the fuzzy model are classified.

Example 2.

1. For families $\mathcal{A}(x)$ and $\mathcal{B}(y)$ classified by three fuzzy attributes, $A_{i}, B_{j}, i, j=1,2,3$ whose characteristic functions have the following values $\chi_{x}\left(A_{i}\right), \chi_{y}\left(B_{j}\right) \in\{-1,0,1\}$, the families of the base clusters of the evaluated states of the output variable represented with the following set:

$$
[K]^{0}=\left\{[-2]^{0},[-1]^{0},[0]^{0},[1]^{0},[2]^{0}\right\} .
$$

2. For families $\mathcal{A}(x), \mathcal{B}(y)$ classified by five fuzzy attributes whose characteristic functions have the following values $\chi_{x}\left(A_{i}\right), \chi_{y}\left(B_{j}\right) \in\{-2,-1,0,1,2\}$, the family of base clusters of the evaluated states of the output variable is represented with the following set:

$$
[K]^{0}=\left\{[-4]^{0},[-3]^{0},[-2]^{0},[-1]^{0},[0]^{0},[1]^{0},[2]^{0},[3]^{0},[4]^{0}\right\} .
$$

3. If families $\mathcal{A}(x), \mathcal{B}(y)$ are classified by $p$ fuzzy attributes, the families of base clusters of the evaluated states of the output variable generated by pairs $\left(A_{i}, B_{j}\right)$ of fuzzy numbers are represented with the following set:

$$
[K]^{0}=\{0, \pm 1, \pm 2, \ldots, \pm(p-1)\} .
$$

4. Let families $\mathcal{A}(x)$ and $\mathcal{B}(y)$ be classified with an even number of fuzzy attributes, e.g. $A_{i}, B_{j}, i, j=1,2$ whose characteristic functions have the following values $\chi_{x}\left(A_{i}\right), \chi_{y}\left(B_{j}\right) \in\{-1,1\}$ then the family of the base clusters of the evaluated states of the output variable is the following set

$$
[K]^{0}=\left\{[-2]^{0},[0]^{0},[2]^{0}\right\} .
$$

Let the output variable of the model be evaluated by the set of all pairs $\left(A_{i}, B_{j}\right) \in \mathcal{A}(x) \times \mathcal{B}(y)$, and let $\tau_{x}\left(A_{i}\right), \chi_{x}\left(A_{i}\right), \tau_{y}\left(B_{j}\right), \chi_{y}\left(B_{j}\right)$ be the characteristic elements of fuzzy numbers $A_{i} \in \mathcal{A}(x), B_{j} \in \mathcal{B}(y)$, by which the values of input variables $x, y$ are evaluated. To each state $s_{i, j}=\left(A_{i}, B_{j}\right)$ of the output variable of the model there corresponds a real number $k^{\star}$ equal to the value of the characteristic function, and the following is valid

$$
k_{i, j}^{\star}=\chi_{x, y}^{\star}\left(A_{i}, B_{j}\right)=C \cdot\left(l \cdot \chi_{x}\left(A_{i}\right)+\chi_{y}\left(B_{j}\right)\right) .
$$

where $C=\frac{m_{1}+m_{2}}{m_{1} \cdot l+m_{2}}, \mathrm{i} l=\frac{\tau_{x}\left(A_{i}\right)}{\tau_{y}\left(B_{j}\right)}$ is a constant.

Definition 13. Let $\left[k_{i, j}\right]^{0}, k \in \mathbb{Z}$ be a base cluster from the cluster family of $[K]^{0}$ (Figure 4). We consider that state $s_{i, j}=\left(A_{i}, B_{j}\right)$ belongs to base cluster $\left[k_{i, j}\right]^{0}$ if and only if the value of the characteristic function

$$
\chi_{x, y}^{\star}\left(A_{i}, B_{j}\right)=\left[k_{i, j}^{\star}\right], k \in \mathbb{R}
$$

belongs to the base cluster, i.e. if the following is valid

$$
\chi_{x, y}^{\star}\left(s_{i, j}\right)=k_{i, j}^{\star} \in\left[\frac{2 k-1}{2} ; \frac{2 k+1}{2}\right]^{0}=[k]^{0} .
$$

and we write it as $s_{i, j}=\left(A_{i}, B_{j}\right) \in\left[k_{i . j}\right]^{0}$. The states of one cluster describe the equal value of the output variable.

Based on the above, we define the state clustering algorithm which then describes the output variable.

\section{Algorithm for Clustering the Evaluated Values of the Output Variable}

Let us summarize the presented results in the form of an algorithm. For a simpler understanding of the procedure, let us consider a fuzzy model with two input variables and one output variable. Let the input variable $x$ be given by family $\mathcal{A}(x)$ of $p$ fuzzy attribute, and variable $y$ be given by family $\mathcal{B}(y)$ of $q$ fuzzy attribute.

The product:

$$
\begin{aligned}
\mathcal{S} & =\mathcal{A}(x) \times \mathcal{A}(x)=\left\{\left(A_{i}, B_{j}\right) \mid A_{i} \in \mathcal{A}(x), B_{j} \in \mathcal{B}(y)\right\} \\
i & =1,2, \ldots, p ; j=1,2, \ldots, q
\end{aligned}
$$

determines a set of all states which can have their output variable determined. The clustering algorithm of the output state is obtained in the following steps:

1. For each input variable based on the defined terms and formulas, let us determine the characteristic elements as in the following Table 1.

2. Form a matrix of the descriptive states of the output variable determined by the Cartesian product of the families of input variables

$$
\begin{gathered}
M_{u}(\mathcal{S})=\left[s_{i, j}\right]_{p \times q} ; s_{i, j}=\left(A_{i}, B_{j}\right), \\
i=1,2, \ldots, p ; j=1,2, \ldots, q .
\end{gathered}
$$


Based on the results from step $\mathrm{K} 1$, we form a matrix of

$$
i=1,2, \ldots, p ; j=1,2, \ldots, q \text {. }
$$
base clusters

$$
[K]^{0}=\left[\left[k_{i, j}\right]^{0}\right]_{p \times q}, k_{i, j}^{0}=\chi_{x}\left(A_{i}\right)+\chi_{y}\left(B_{j}\right) ;
$$

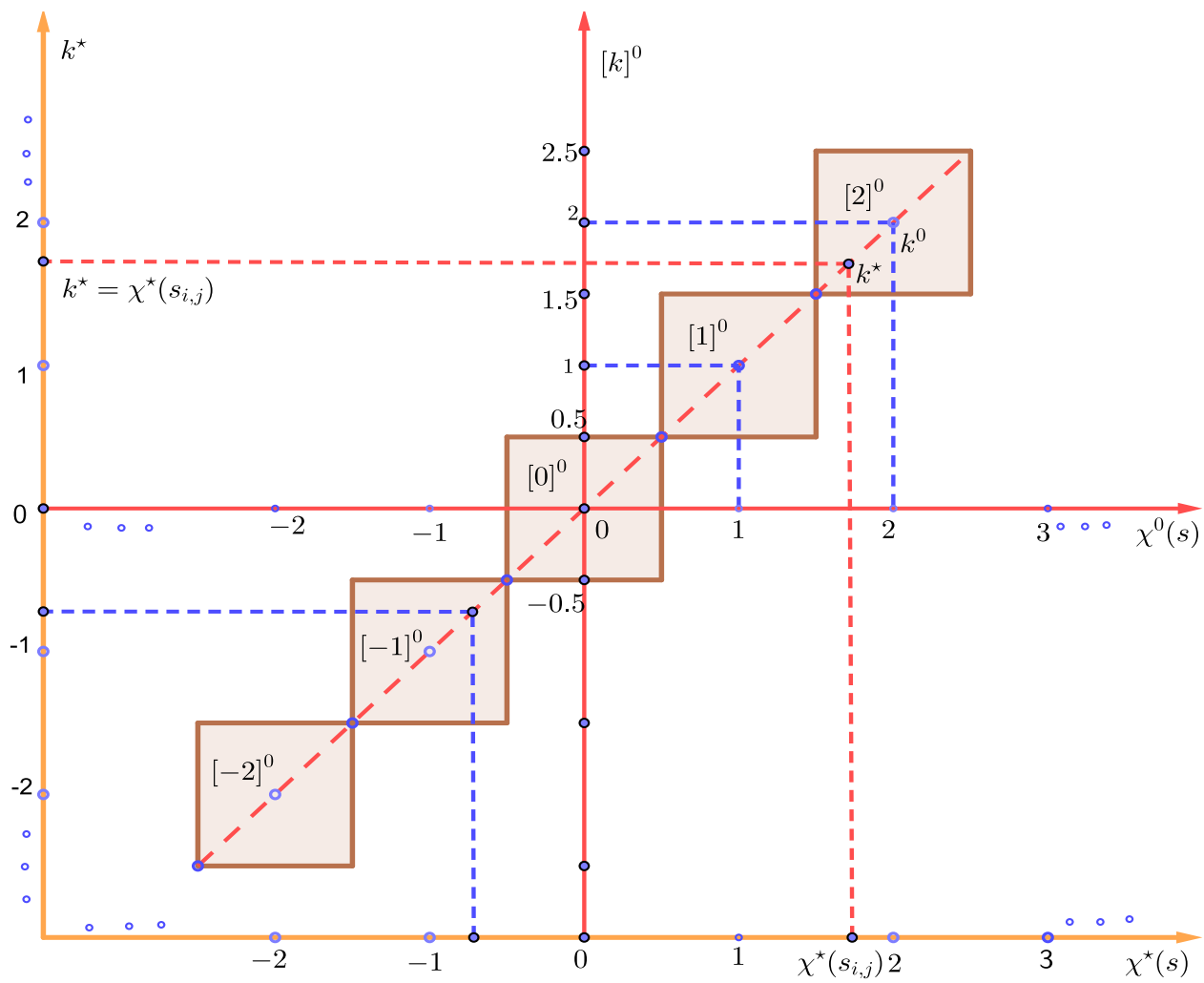

Figure 4. Construction of a cluster diagram of a characteristic function of a pair of fuzzy numbers.

Table 1. Characteristic elements of the input variables.

\begin{tabular}{llllll}
\hline Input Variable & Daf & $\boldsymbol{m}_{2}$ & $\boldsymbol{\tau}$ & $\chi$ & $\boldsymbol{\rho}$ \\
\hline$x$ & $\operatorname{Daf}\left(A_{1}\right)$ & $m_{2}^{1}$ & $\tau_{x}\left(A_{1}\right)$ & $\chi_{x}\left(A_{1}\right)$ & $\rho_{x}\left(A_{1}\right)$ \\
& $\operatorname{Daf}\left(A_{2}\right)$ & $m_{2}^{2}$ & $\tau_{x}\left(A_{2}\right)$ & $\chi_{x}\left(A_{2}\right)$ & $\rho_{x}\left(A_{2}\right)$ \\
& $\ldots$ & & & & \\
& $\operatorname{Daf}\left(A_{p}\right)$ & $m_{2}^{p}$ & $\tau_{x}\left(A_{p}\right)$ & $\chi_{x}\left(A_{p}\right)$ & $\rho_{x}\left(A_{p}\right)$ \\
$y$ & $\operatorname{Daf}\left(B_{1}\right)$ & $m_{2}^{1}$ & $\tau_{y}\left(B_{1}\right)$ & $\chi_{y}\left(B_{1}\right)$ & $\rho_{y}\left(B_{1}\right)$ \\
& $\operatorname{Daf}\left(B_{2}\right)$ & $m_{2}^{2}$ & $\tau_{y}\left(B_{2}\right)$ & $\chi_{y}\left(B_{2}\right)$ & $\rho_{y}\left(B_{2}\right)$ \\
& $\ldots$ & & & & \\
& $\operatorname{Daf}\left(B_{q}\right)$ & $m_{2}^{q}$ & $\tau_{y}\left(B_{q}\right)$ & $\chi_{y}\left(B_{q}\right)$ & $\rho_{y}\left(B_{q}\right)$ \\
\hline
\end{tabular}

3. Using the results from $\mathrm{K} 1$ and the formula for the weighted mean and the weight impact of the pair of fuzzy numbers, we then determine the matrices of the characteristic elements of the state $s$, as follows:

$$
\tau_{x, y}(\mathcal{S})=\left[\tau_{x, y}\left(s_{i, j}\right)\right]_{p \times q} ; \rho_{x, y}(\mathcal{S})=\left[\rho_{x, y}\left(s_{i, j}\right)\right]_{p \times q}
$$

4. For the calculated characteristic values of state $\chi_{x, y}^{\star}\left(s_{i, j}\right)$ we form a matrix $\left[K^{\star}\right]=\left[\chi_{x, y}^{\star}\left(s_{i . j}\right)\right]_{p \times q}=\left[k_{i, j}^{\star}\right]_{p \times q}$ using the following formula

$$
k_{i, j}^{\star}=\chi_{x, y}^{\star}\left(A_{i}, B_{j}=\frac{m_{1}+m_{2}}{m_{1} \cdot l+m_{2}}\left(l \cdot \chi_{x}\left(A_{i}\right)+\chi_{y}\left(B_{j}\right)\right)\right.
$$

5. By comparing matrix $\left[K^{\star}\right]$ of state cluster $s \in \mathcal{S}$ with matrix $[K]^{0}$ of base clusters s, i.e. $\left[K^{\star}\right] \approx[K]^{0}$, we determine how the states are distributed in relation to the base clusters, that is, we determine the elements of each base cluster $[k]^{0} \in[K]^{0}$.

\section{Characteristic Elements of n-Fuzzy Numbers}

The terms defined in the case of models with two input variables are similarly defined also in the case of models with $n$ input variables. Let variables $x_{1}, x_{2}, \ldots, x_{n}$ be classified by families $\mathcal{A}_{1}, \mathcal{A}_{2}, \ldots, \mathcal{A}_{n}$ with corresponding intervals of dominant affiliation $D a f_{x_{1}}\left(\mathcal{A}_{1}\right), D a f_{x_{2}}\left(\mathcal{A}_{2}\right), \ldots, D a f_{x_{n}}\left(\mathcal{A}_{n}\right)$, then all states $s=\left(A_{1}, A_{2}, \ldots, A_{n}\right)$ of the output variable are determined by the Cartesian product $\mathcal{A}_{1} \times \mathcal{A}_{2} \times \ldots \times \mathcal{A}_{n}$

The value of the weighted means of $\mathrm{n}$-fuzzy numbers $\left(A_{1}, A_{2}, \ldots, A_{n}\right)$ on n-dimensional square (simplex) of the dominant affiliation is defined by the relation:

$$
\tau_{x_{1}, x_{2}, \ldots, x_{n}}\left(A_{1}, A_{2}, \ldots, A_{n}\right)=\frac{\sum_{i=1}^{n} m_{i} \tau_{x_{i}}\left(A_{i}\right)}{\sum_{i=1}^{n} m_{i}}
$$

where $m_{i}=\frac{a_{i-1}+a_{i}}{2}$ is the corresponding mean of the interval of dominant affiliation of fuzzy number $A_{i}, i=1,2, \ldots, n$, and $\tau_{x}\left(A_{i}\right)$ is the weighted value of the mean of fuzzy number $A_{i}$ on the interval of dominant affiliation.

The function of the weight impact of the n-number of 
fuzzy numbers $\left(A_{1}, A_{2}, \ldots, A_{n}\right) \in \mathcal{A}_{1} \times \mathcal{A}_{2} \times \ldots \times \mathcal{A}_{n}$ by which variables $x_{1}, x_{2}, \ldots, x_{n}$ on the n-dimensional square (simplex) are evaluated, with the dominant affiliation of fuzzy numbers $A_{1}, A_{2}, \ldots, A_{n}$, is defined in a relation:

$$
\begin{gathered}
\rho_{x_{1}, x_{2}, \ldots, x_{n}}\left(A_{1}, A_{2}, \ldots, A_{n}\right)=\sum_{i=1}^{n} \rho_{x_{i}}\left(A_{i}\right) \\
=\sum_{i=1}^{n} \chi_{x_{i}}\left(A_{i}\right) \tau_{x_{i}}\left(A_{i}\right)
\end{gathered}
$$

The characteristic function of the influence of n-fuzzy numbers $\left(A_{1}, A_{2}, \ldots, A_{n}\right) \in \mathcal{A}_{1} \times \mathcal{A}_{2} \times \ldots \times \mathcal{A}_{n}$ is defined in the following relation

$$
\chi\left(A_{1}, A_{2}, \ldots, A_{n}\right)=\frac{\sum_{i=1}^{n} \chi_{x_{i}}\left(A_{i}\right) \tau_{x_{i}}\left(A_{i}\right)}{\tau\left(A_{1}, A_{2}, \ldots, A_{n}\right)}=\frac{\rho_{x_{1}, x_{2}, \ldots, x_{n}}\left(A_{1}, A_{2}, \ldots, A_{n}\right)}{\tau\left(A_{1}, A_{2}, \ldots, A_{n}\right)}
$$

All other terms are also defined analogously, as in the case of a pair of fuzzy numbers.

\section{Application of Classification Algori- thm in Evaluating the Economic Dimension of Sustainability of Tourism Development}

A sustainable model of tourism development is a model that is considered to be sustainable in every segment of its impact, from economic, socio-cultural and environmental impact to the impact on tourist satisfaction. To evaluate the impact of tourism on individual segments of activity, we use indicators that represent this impact. Let us analyze how the impact of tourism is measured by comparative indicators from the aspect of optimizing the goals of sustainable tourism development in a given destination. For each of the proposed comparative indicators, we introduce symbols and express them in a certain relationship, when possible, in order to formulate a simple mathematical model that would express the dependence of the sustainability of tourism development in a given destination on the goals that are the basis for its implementation. [1, 9, 10, 12]

\subsection{Economic Effects of Tourist Activity on a Given Destination}

The following indicators have been proposed to measure the economic effects of tourism in a destination:

1) Seasonality of traffic - the percentage of visits to a destination in the full tourist season (lasting 3 months) in relation to the annual visits,

2) Relation between the number of overnight stays and accommodation capacities of a destination or the ones available in its surroundings (rural households),

3) Coefficient of local tourist increase not yet determined.

In relation to the value of the visit rate - seasonality of the visit to a destination realized during the season (three months) (calculated by the formula; $s=\frac{n_{s}}{n_{g}}$, where $n_{s}$ is a number of visitors in a season, $n_{g}$ is a number of visitors during the year) the economic effects are evaluated as follows

$$
s=\left\{\begin{array}{lll}
\text { sustainable } & \text { if } & 0<s<0.4 \\
\text { tolerable } & \text { if } & 0.4 \leq s<0.5 \\
\text { unsustainable } & \text { if } & 0.5 \leq s \leq 1
\end{array}\right.
$$

Similarly, based on the value of the indicator of the ratio of the number of overnight stays and accommodation capacities (calculated by the formula; $k=\frac{n_{n}}{n_{s}}$ where $n_{n}$ is a number of overnight stays in the season, $n_{s}$ is a number of accommodation capacities of the destination and its surroundings), we measure the utilization of these capacities. If the value of this ratio is less than 120 of overnight stays per bed per year, it is considered that the degree of utilization of accommodation capacity is economically unsustainable; if the value of ratio $k$ is between 120 and 150 overnight stays per bed, the degree of utilization of accommodation capacity is considered to be tolerable; and for the value of ratio greater than 150 nights per bed per year, the accommodation capacities are considered to be economically viable. The dependence of the state of economic viability on the degree of utilization of accommodation capacities expressed as a percentage can be written as follows:

$$
k=\left\{\begin{array}{lll}
\text { sustainable } & \text { if } & k>0.42 \\
\text { tolerable } & \text { if } & 0.33 \leq k<0.42 \\
\text { unsustainable } & \text { if } & k \leq 0.33
\end{array}\right.
$$

The indicator of the evaluation of the impact of tourism on local economic growth - let us mark it with $l$ - does not have a precisely defined threshold value. Due to the complexity of the criterion itself, it is not easy to precisely determine the limits of significance of its impact, but it is left to the expert to assess the value of the flow indicator, depending on the goal and the need for measurement. Let us leave the value of coefficient $l$ as an open question, as it is, and for this occassion of quantifying the economic effects of tourism development, let us assume that the importance of the development of tourism in the local economy can be evaluated as follows

$$
l=\left\{\begin{array}{lll}
\text { impact is significat and growing } & \text { if } & l \geq 0.67 \\
\text { impact is tolerable and changing } & \text { if } & 0.33 \leq l \leq 0.67 \\
\text { impact is insignificant and declining } & \text { if } & l \leq 0.33
\end{array}\right.
$$

Based on the obtained values of the indicators $s, k, l$, we monitor the economic benefits achieved by tourist entities at the destination and the local community and the local population from the development of tourism. Let us express this relationship by the following relation, $E=f_{1}(s, k, l)+$ $\mu_{1}$, where $E$ denotes the value of economic benefit from the development of tourism in the destination, and $f_{1}$ is the functional relationship of indicators and economic profits, and $\mu_{1}$ is the error of assessment of the expressed economic benefit as assessed by indicators $s, k, l$. Every economic activity, including tourism, aims to maximize its economic effects. Therefore, the goal of the tourism industry, accompanying economic entities at the destination and in the surroundigs is to maximize the economic effects of tourism, that is to maximize the function, which can be written in the 
following form

$$
\max E=\max _{s<0.4 \wedge k>0.42 \wedge l \geq 0.67} f_{1}(s, k, l)+e_{1} .
$$

\subsection{Fuzzification of Input Variable Models}

The input variables in the process of evaluating the sustainability of tourism development are indicators for assessing the value of the dimensions of the impact of tourism development, which we have marked with $E, S I, K I, S R, Z$, and the output variable is the assessed sustainability of tourism development, which we have marked with $O_{R}$. In order to quantitatively assess the economic viability of tourism development at a given destination, let us establish a link between the fuzzy numbers and indicators of the evaluation of the impact of tourism development. [9, 10, 12]

All values of indicators by which we evaluate the impact of tourism are expressed by a certain ratio. Let us denote this relation by $P$, and let us put that $P=\frac{M}{N}$ and $P \in[0,1]$, where $M, N$ are the measured quantities, which we have defined in the previous part. Note that the recommended limit values in which the values of the measured indicators need to be found divide segment $[0,1]$ into disjoint subintervals. In relation to the determined value of $P$, the state of sustainability of tourism development in relation to the observed dimension, as measured by the appropriate indicator is described with the following attributes: sustainable, unsustainable, tolerable which represent one indicator, over the quantitative domain $U=[0,1]$ divided by points $a_{1}, a_{2}$ into disjoint intervals, and evaluated by the family of fuzzy sets (Figure 5).

$$
\mathcal{A}(x)=\{\text { state is unsustainable, state is tolerable, }
$$

$$
\text { state is sustainable }\}=\{N, P, O\}
$$

where $N=$ state is unsustainable,

$P=$ state is tolerable, and $O=$ state is sustainable, with domains of value $U_{N}, U_{P}, U_{O} \subseteq U=[0 ; 1]$ respectively.

Using the defined limit values of comparative indicators by which we measure the sustainability of tourism development, let us now form the functions of affiliation of the given fuzzy numbers by which the indicators were evaluated.

For indicators evaluated by three triangular fuzzy numbers $N, P, O$ and whose fuzzy number has the following designations: $P=$ state is tolerable, segment $\left[a_{1}, a_{2}\right]=$ $[0.33 ; 0.67]$ interval of dominant affiliation, there are two possibilities of ordering the indicator evaluation based on the corresponding intervals of dominant affiliations, namely:

1. when $\operatorname{Daf}_{x}(N)=\left[0, a_{1}\right]=[0 ; 0.33] \subset U_{N}$ fuzzy number $N=$ state is unsustainable, and $\operatorname{Da} f_{x}(0)=\left[a_{2}, 1\right]=$ $[0.67 ; 1] \subset U_{O}$ and the interval of dominant ffiliation to fuzzy number $O=$ state is sustainable, and the case when

2. when $\operatorname{Daf}_{x}(O)=\left[0, a_{1}\right] \subset U_{O}$ the interval of dominant affiliation to fuzzy number $O=$ state is sustainable, and $\operatorname{Daf}_{x}(N)=\left[a_{2}, 1\right] \subset U_{N}$ is the interval of dominant affiliation to fuzzy number $N=$ state is unsustainable, (Figure 5). Hence, the functions of affiliation to fuzzy numbers $M P=$ less than tolerable, $P=$ state is tolerable and $V P=$ higher than tolerable, where fuzzy numbers $M P, V P$ correspond to fuzzy numbers $M P=N=$ state is unsustainable,$\quad$ i.e. $V P=O=$ state is sustainable, depending on whether it is the case of (1) or case (2) according to the defined limit value of indicator $x$, and they read as follows (Figure 5)

$$
\begin{gathered}
\mu_{M P}(x)= \begin{cases}1, & x=0 \\
1-x, & 0 \leq x \leq 1\end{cases} \\
\mu_{P}(x)= \begin{cases}2 x, & 0 \leq x \leq 0.5 \\
2(1-x), & 0.5 \leq x \leq 1\end{cases} \\
\mu_{V P}(x)= \begin{cases}1, & x=1 \\
x, & 0 \leq x \leq 1\end{cases}
\end{gathered}
$$

For indicators evaluated by three fuzzy numbers:

1. trapezoidal fuzzy number $P=$ state is tolerable, with interval of dominant affiliation $\operatorname{Daf}_{x}(P)=\left[c_{1}, c_{2}\right] \subset U_{P}$, kernel $\operatorname{Ker}(P)=\left[b_{1} ; b_{2}\right]$ and supplier $\operatorname{supp}(P)=\left\langle a_{1}, a_{2}\right\rangle$ and fuzzy numbers

2. $M P=$ less than tolerable, with interval of dominant affiliation $D a f_{x}(M P)=\left[0 ; c_{1}\right]$, kernel $\operatorname{Ker}(M P)=\left[0, a_{1}\right]$ and supplier $\operatorname{supp}(M P)=\left[0, b_{1}\right\rangle$ and

3. $V P 1=$ higher than tolerable, with intervals of dominant affiliation $\operatorname{Da} f_{x}(V P)=\left[c_{2}, 1\right]$, kernel $\operatorname{Ker}(V P)=$ $\left[a_{2}, a\right]$ and supplier $\operatorname{supp}(V P)=\left\langle b_{2}, 1\right]$, depending on whether it is the case of (1) or case (2), the functions of affiliation are ordered as follows (Figure 5)

$$
\begin{gathered}
\mu_{M P}(x)= \begin{cases}1, & 0 \leq x \leq a_{1} \\
\frac{b_{1}-x}{b_{1}-a_{1}}, & a_{1} \leq x \leq b_{1}\end{cases} \\
\mu_{V P}\left(= \begin{cases}1, & a_{2} \leq x \leq 1 \\
\frac{x-b_{2}}{a_{2}-b_{2}}, & b_{2} \leq x \leq a_{2}\end{cases} \right. \\
\mu_{P}(x)= \begin{cases}\frac{x-a_{1}}{b_{1}-a_{1}}, & a_{1} \leq x \leq b_{1} \\
\frac{a_{2}-x}{a_{2}-b_{2}}, & b_{1} \leq x \leq b_{2}\end{cases}
\end{gathered}
$$

where $b_{1}=\frac{a_{1}}{1-a_{1}}$, and $b_{2}=2-\frac{1}{a_{2}}$ are the limits of the kernel of trapezoidal fuzzy number $P$, i.e. segment $\operatorname{Ker}(P)=$ $\left[b_{1}, b_{2}\right]=\left[\frac{a_{1}}{1-a_{1}} ; 2-\frac{1}{a_{2}}\right]$. 

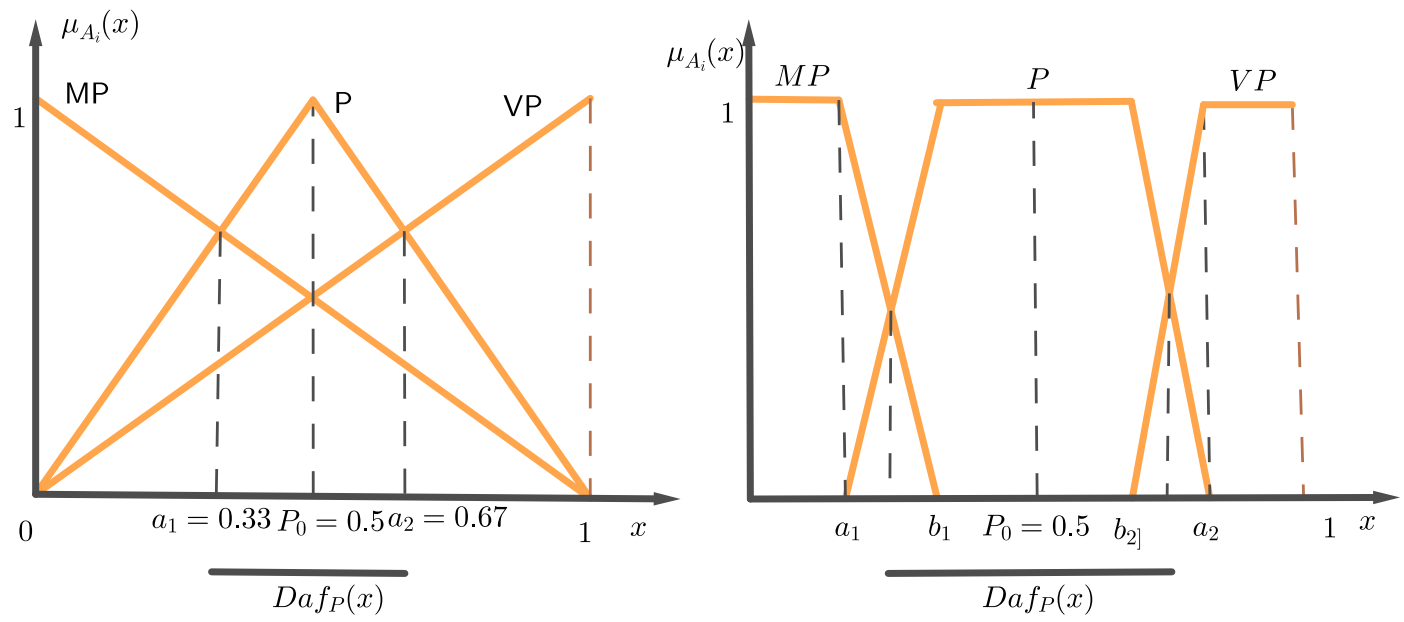

Figure 5. Construction of triangular and trapezoidal fuzzy numbers and their basic elements.

When the limits of kernel $\operatorname{Ker}(P)=\left[b_{1}, b_{2}\right]$ of trapezoidal fuzzy number $P$ are known, then the limits of its supplier and the intervals of dominant affiliation are calculated by the following formulas

$$
a_{1}=\frac{b_{1}}{1+b_{1}}, a_{2}=\frac{1}{2-b_{2}}, c_{1}=\frac{a_{1}+b_{1}}{2}, c_{2}=\frac{a_{2}+b_{2}}{2}
$$

\subsection{Modeling the Input-Output Variable Models}

\subsubsection{Modeling the Input Variable Model}

Based on the constructed fuzzy numbers and limit values of indicators for measuring the sustainability of tourism development, let us complete the fuzzification of comparative indicators as input variables in the fuzzy model using their recommended limit values.[6-8]

1. Let us denote the linguistic variable of seasonality of visit by $x$ and write it in the form of $\left(x, \mathcal{S}(x), \mathcal{U}_{1}, M_{1}\right)$. The domain of values that variable $x$ can take is defined by the family of fuzzy sets:

$$
\begin{aligned}
& \mathcal{S}(x)=\{\text { state is sustainable, state is tolerable, } \\
& =\left\{\left(x_{i}, \mu_{S_{i}}(x)\right) \mid x \in S_{i}, S_{i} \subset \mathcal{U}_{1} \subseteq[0 ; 1], i=1,2,3\right\}=\left\{S_{0}, S_{P}, S_{N}\right\},
\end{aligned}
$$

where the functions of the fuzzy numbers $S_{0}, S_{P}, S_{N}$ are written in the form of a triangular, trapezoidal or $\Gamma$ fuzzy number;

The functions of affiliation to fuzzy sets $S_{0}, S_{P}, S_{N}$, with intervals of dominant affiliation, by which the indicator of the seasonality of visit is evaluated, are written in the trapezoidal form, and they are:

a) For fuzzy number $S_{O}=$ state is sustainable, a shorter record of the function of affiliation is $S_{O}=$ trapm $f[0,0,0.286,0.4]$ and $D a f_{x}\left(S_{O}\right)=[0,0.34]$

b) For fuzzy number $S_{P}=$ state is tolerable, a shorter record of the function of affiliation is

c) $S_{P}=\operatorname{Trapm} f[0.286,0.4,0.5,0.667]$ and $\operatorname{Da} f_{x}\left(S_{P}\right)=$ $[0.34,0.58]$

d) For fuzzy number $S_{N}=$ state is unsustainable, a shorter record of the function of affiliation is $S_{N}=$ trapm $f[0.5,0.667,1,1]$ and $D a f_{x}\left(S_{N}\right)=[0.58,1]$.

2. Let us denote the linguistic variable of utilization of accommodation capacities by $y$ and write it in the form of $\left(y, \mathcal{K}(y), \mathcal{U}_{2}, M_{2}\right)$. Let $\mathcal{K}$ be a set of all fuzzy numbers which will describe the linguistic variable utilization of accommodation capacities through the ratio of the number of overnight stays and accommodation capacities available at a given destination. The domain of the value of variable $y$ is defined by the family of fuzzy sets

$$
\begin{gathered}
\mathcal{K}(y)=\{\text { state is sustainable, state is tolerable, } \\
\text { state is unsustainable }\} \\
=\left\{\left(y_{i}, \mu_{K_{i}}(y)\right) \mid y \in K_{i}, K_{i} \subset \mathcal{U}_{2} \subseteq[0 ; 1], i=1,2,3\right\} \\
=\left\{K_{0}, K_{P}, K_{N}\right\},
\end{gathered}
$$

where fuzzy numbers $K_{0}, K_{P}, K_{N}$ are defined as follows:

a) fuzzy number $K_{N}=\operatorname{trapm} f[0,0,0.25,0.33]$ and $\operatorname{Daf}_{y}\left(K_{O}\right)=[0.0 .29]$

b) fuzzy number $K_{P}=\operatorname{trapm} f[0.25,0.33,0.42,0.63]$, and $\operatorname{Daf}_{y}\left(K_{P}\right)=[0.29,0.53]$

c) fuzzy number $K_{O}=\operatorname{trapm} f[0.42,0.63,1,1]$ and $\operatorname{Daf} f_{y}\left(K_{N}\right)=[0.53,1]$.

Let us denote the linguistic variable of impact of tourism on the local economy by $z$ and write it in the form of $\left(z, \mathcal{L}(z), \mathcal{U}_{3}, M_{3}\right)$. The domain of the value of variable $z$ is defined by the family of fuzzy sets $L(z)=\{$ the impact is significant and growing, the impact is tolerably variable, the impact is insignificant and declining

$$
=\left\{\left(z_{i}, \mu_{L_{i}}(z)\right) \mid z \in L_{i}, L_{i} \subset \mathcal{U}_{3} \subseteq[0 ; 1], i=1,2,3\right\}=\left\{L_{0}, L_{P}, L_{N}\right\},
$$

where fuzzy numbers $L_{0}, L_{P}, L_{N}$ are defined by triangular functions of affiliation, as follows

a) fuzzy number $L_{N}=\operatorname{trim} f[0,0,1], \operatorname{Da} f_{z}\left(L_{N}\right)=[0,0.33]$.

b) fuzzy number $L_{P}=\operatorname{trimf}[0,0.5,1], \operatorname{Daf} f_{z}\left(L_{P}\right)=$ $[0.33,0.67]$

c) fuzzy number $L_{O}=\operatorname{trimf}[0,1,1], \operatorname{Daf} f_{z}\left(L_{O}\right)=[0.67,1]$.

\subsubsection{Modeling the Input Variable Models}

Let the linguistic variable of the economic value of tourism development be denoted $\left(e, \mathcal{E}, \mathcal{U}_{E}, M_{E}\right)$ and let it have the values on universal set $U_{E}=[0,1]$ and let it be evaluated with five qualitative states, i.e. the values that variable $e$ can take, and let us now define it by the family of fuzzy sets 
$\mathcal{E}(e)=\{$ state is alarming, state is intolerable, state is tolerable, state is good, state is sustainable $\}=$

$$
\left\{E_{A}, E_{N}, E_{P}, E_{D}, E_{O}\right\}
$$

$=\left\{\left(e, \mu_{E_{i}}(e)\right) \mid e=(x, y, z) \in E_{i}, E_{i} \subset \mathcal{U}_{E} \subseteq[0 ; 1], i=A, N, P, O\right\}$. where fuzzy numbers individually have the meanings as in Table 2.

The functions of affiliation to fuzzy sets

$E_{A}, E_{N}, E_{P}, E_{D}, E_{O}$ with intervals of dominant affiliation, by which the economic value of the sustainability of tourism development were evaluated are shown in (Figure 6).

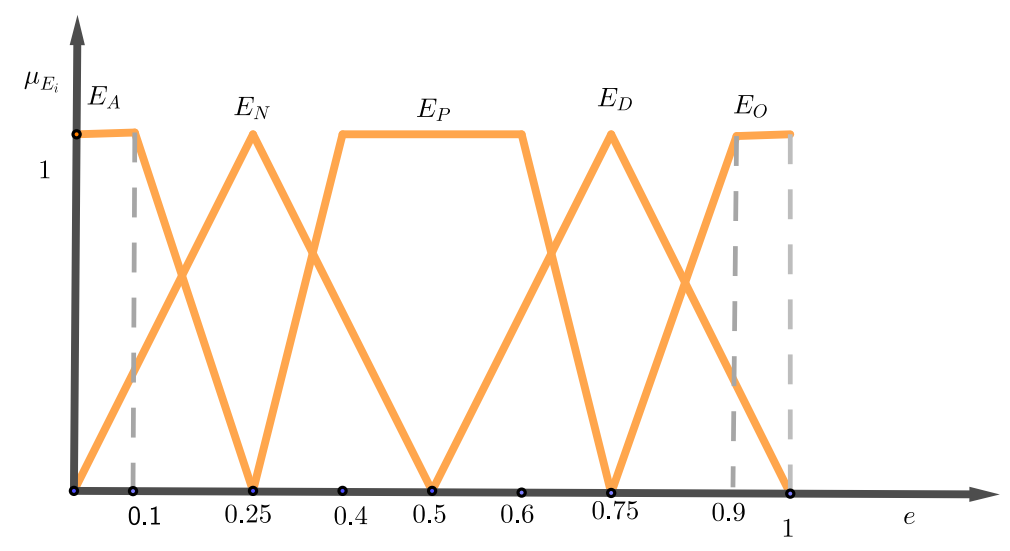

Figure 6. Functions of affiliation to a family of fuzzy numbers of the variable economic value of tourism development.

The functions of affiliation of fuzzy numbers, by which the quantitative state of the economic effects of tourism development was described, are given by the following equations, and with the interval of dominant affiliation, as follows:

1. For fuzzy number $E_{A}$ it is

$$
\mu_{E_{A}}(e)=\left\{\begin{array}{lll}
1 & \text { for } & 0 \leq e \leq 0.1 \\
\frac{0.25-e}{0.15} & \text { for } & 0.1 \leq e \leq 0.25
\end{array}\right.
$$

we write it shorter in the following form:

$$
E_{A}=\operatorname{trapp} f[0 ; 0 ; 0.1 ; 0.25], \operatorname{Daf}\left(E_{A}\right)=[0 ; 0.156] .
$$

2. For fuzzy number $E_{N}$ it is

$$
\mu_{E_{N}}(e)=\left\{\begin{array}{lll}
\frac{e}{0.25} & \text { for } & 0 \leq e \leq 0.25 \\
\frac{0.5-e}{0.25} & \text { for } & 0.25 \leq e \leq 0.5
\end{array}\right.
$$

we write it shorter in the following form: $E_{N}=$ triang $f[0 ; 0.25 ; 0.5], \operatorname{Daf}\left(E_{N}\right)=[0.156 ; 0.344]$.

3. For fuzzy number $E_{P}$ it is

$$
\mu_{E_{P}}(e)=\left\{\begin{array}{lll}
\frac{e-0.25}{0.15} & \text { for } & 0.25 \leq e \leq 0.40 \\
1 & \text { for } & 0.4 \leq e \leq 0.6 \\
\frac{0.75-e}{0.15} & \text { for } & 0.6 \leq e \leq 0.75
\end{array}\right.
$$

we write it shorter in the following form: $E_{P}=$ trapp $f[0.25 ; 0.4 ; 0.6 ; 0.75], \operatorname{Daf}\left(E_{P}\right)=[0.344 ; 0.656]$.

4. For fuzzy number $E_{D}$ it is

$$
\mu_{E_{D}}(e)=\left\{\begin{array}{lll}
\frac{e-0.5}{0.25} & \text { for } & 0.5 \leq e \leq 0.75 \\
\frac{1-e}{0.25} & \text { for } & 0.75 \leq e \leq 1
\end{array}\right.
$$

we write it shorter in the following form:

$E_{D}=\operatorname{triang} f[0.5 ; 0.75 ; 1], \operatorname{Daf}\left(E_{D}\right)=[0.656 ; 0.844]$.

5. For fuzzy number $E_{O}$ it is

$$
\mu_{E_{P}}(e)=\left\{\begin{array}{lll}
\frac{e-0.75}{0.15} & \text { for } & 0.75 \leq e \leq 0.90 \\
1 & \text { for } & 0.9 \leq e \leq 1
\end{array}\right.
$$

we write it shorter in the following form:

$$
E_{O}=\operatorname{trapp} f[0.75 ; 0.9 ; 1 ; 1], \operatorname{Daf}\left(E_{O}\right)=[0.844 ; 1] .
$$

Table 2. Quantitative significance of fuzzy numbers which describe the assessed state of the impact of tourism development on the local economy.

\begin{tabular}{ll}
\hline Quantitative state & $\begin{array}{l}\text { Significance of the economic effects of tourism } \\
\text { development is: }\end{array}$ \\
\hline$E_{A} \equiv$ alarming & completely unsustainable \\
$E_{N} \equiv$ intolerable & weak and change is needed \\
$E_{P} \equiv$ tolerable & tolerable and can be improved \\
$E_{D} \equiv$ good & good and show tendency towards sustainability \\
$E_{O} \equiv$ sustainable & sustainable \\
\hline
\end{tabular}

\subsection{Clustering the States of the Output Variable Model}

The domain of features which describe the impact of tourism activity on the economic dimension $E$ is determined by the product of the domain of features of the measured indicators. Thus, the product $E=\mathcal{S} \times \mathcal{K} \times \mathcal{L}$ represents a set of all fuzzy sets which describe all possible states of the tourism development economic impact evaluation measured by indicators $S, K, L$. That is, the possible states are: 


$$
\begin{aligned}
E=S \times & K \times L=\left\{S_{O}, S_{P}, S_{N}\right\} \times\left\{K_{N}, K_{P}, K_{O}\right\} \times\left\{L_{N}, L_{P}, L_{O}\right\} \\
= & \left\{\left(S_{O}, K_{N}, L_{N}\right),\left(S_{O}, K_{N}, L_{P}\right),\left(S_{O}, K_{N}, L_{O}\right)\right. \\
& ,\left(S_{O}, K_{P}, L_{N}\right),\left(S_{O}, K_{P}, L_{P}\right),\left(S_{O}, K_{P}, L_{O}\right) \\
& ,\left(S_{O}, K_{O}, L_{N}\right),\left(S_{O}, K_{O}, L_{P}\right),\left(S_{O}, K_{O}, L_{O}\right) \\
& ,\left(S_{P}, K_{N}, L_{N}\right),\left(S_{P}, K_{N}, L_{P}\right),\left(S_{P}, K_{N}, L_{O}\right) \\
& ,\left(S_{P}, K_{P}, L_{N}\right),\left(S_{P}, K_{P}, L_{P}\right),\left(S_{P}, K_{P}, L_{O}\right) \\
& ,\left(S_{P}, K_{O}, L_{N}\right),\left(S_{P}, K_{O}, L_{P}\right),\left(S_{P}, K_{O}, L_{O}\right) \\
& ,\left(S_{N}, K_{N}, L_{N}\right),\left(S_{N}, K_{N}, L_{P}\right),\left(S_{N}, K_{N}, L_{O}\right) \\
& ,\left(S_{N}, K_{P}, L_{N}\right),\left(S_{N}, K_{P}, L_{P}\right),\left(S_{N}, K_{P}, L_{O}\right) \\
& \left.,\left(S_{N}, K_{O}, L_{N}\right),\left(S_{N}, K_{O}, L_{P}\right),\left(S_{N}, K_{P}, L_{O}\right)\right\}
\end{aligned}
$$

We write the product in the short form as follows $\mathcal{E}=S \times K \times L=\left\{s \mid s=\left(S_{i}, K_{j}, L_{k}\right)\right\}$

where $S$ is the evaluated state of the economic dimension i, $S_{i} \in \mathcal{S}, K_{j} \in \mathcal{K}, L_{k} \in \mathcal{L}, i, j, k \in\{N, P, O\}$.

\subsection{Matrices of Features of Input Variable Models}

Using the defined features of the input variables in the

\begin{tabular}{|c|c|c|c|c|c|}
\hline Designation & Fuzzy number & $\operatorname{Daf}\left(A_{i}\right)$ & $\mathbf{X}\left(\mathbf{A}_{\mathrm{i}}\right)$ & $\tau\left(\mathbf{A}_{\mathbf{i}}\right)$ & $\rho\left(\mathbf{A}_{\mathbf{i}}\right)$ \\
\hline $\mathrm{S}_{0}$ & Trapmf[0,0,0.286,0.4] & {$[0,0.34]$} & 1 & 0.33 & 0.33 \\
\hline $\mathrm{S}_{\mathrm{p}}$ & trapmf $[0.286,0.4,0.5,0.667]$ & {$[0.34,0.58]$} & 0 & 0.32 & 0 \\
\hline $\mathrm{S}_{\mathrm{N}}$ & $\operatorname{trapmf}[0.5,0.667,1,1]$ & {$[0.58,1]$} & -1 & 0.33 & -0.33 \\
\hline $\mathrm{K}_{\mathrm{N}}$ & $\operatorname{trapmf}[0,0,0.25,0.33]$ & {$[0,0.29]$} & -1 & 0.33 & -0.33 \\
\hline $\mathrm{K}_{\mathrm{p}}$ & $\operatorname{trapmf}[0.25,0.33,0.42,0.63$ & {$[0.29,0.53]$} & 0 & 0.26 & 0 \\
\hline $\mathrm{K}_{\mathrm{o}}$ & trapmf $[0.42,0.63,1,1]$ & {$[0.53,1]$} & 0 & 0.33 & 0.33 \\
\hline $\mathrm{L}_{\mathrm{N}}$ & $\operatorname{trimf}[0,0,1]$ & {$[0,0.33]$} & -1 & 0.32 & -0.32 \\
\hline $\mathrm{L}_{\mathrm{p}}$ & $\operatorname{trimf}[0,0.5,1]$ & {$[0.33,0.67]$} & 0 & 0.29 & 0 \\
\hline $\mathrm{L}_{\mathrm{o}}$ & $\operatorname{trimf}[0,1,1]$ & {$[0.67,1]$} & 1 & 0.28 & 0.28 \\
\hline
\end{tabular}
fuzzy model and the rules by which they are determined, let us calculate the characteristic values of indicators which evaluate the economic effects of tourism activities and the obtained values, as shown in Table 3, and let us determine the characteristic values of the states determined by ordered triples $\left(S_{i}, K_{j}, L_{k}\right)$. Let us first determine the matrix of the weighted means of these states, and then the matrix of weight impacts, using the the stated rules.

Table 3. Characteristic elements of fuzzy numbers indicators for evaluating the economic sustainability of tourism development.

$$
\tau_{e}(\mathcal{E})=\left[\begin{array}{lll}
\tau\left(S_{O}, K_{N}, L_{N}\right) & \tau\left(S_{O}, K_{N}, L_{P}\right) & \tau\left(S_{O}, K_{N}, L_{O}\right) \\
\tau\left(S_{O}, K_{P}, L_{N}\right) & \tau\left(S_{O}, K_{P}, L_{P}\right) & \tau\left(S_{O}, K_{P}, L_{O}\right) \\
\tau\left(S_{O}, K_{O}, L_{N}\right) & \tau\left(S_{O}, K_{O}, L_{P}\right) & \tau\left(S_{O}, K_{O}, L_{O}\right) \\
\tau\left(S_{P}, K_{N}, L_{N}\right) & \tau\left(S_{P}, K_{N}, L_{P}\right) & \tau\left(S_{P}, K_{N}, L_{O}\right) \\
\tau\left(S_{P}, K_{P}, L_{N}\right) & \tau\left(S_{P}, K_{P}, L_{P}\right) & \tau\left(S_{P}, K_{P}, L_{O}\right) \\
\tau\left(S_{P}, K_{O}, L_{N}\right) & \tau\left(S_{P}, K_{O}, L_{P}\right) & \tau\left(S_{P}, K_{O}, L_{O}\right) \\
\tau\left(S_{N}, K_{N}, L_{N}\right) & \tau\left(S_{N}, K_{N}, L_{P}\right) & \tau\left(S_{N}, K_{N}, L_{O}\right) \\
\tau\left(S_{N}, K_{P}, L_{N}\right) & \tau\left(S_{N}, K_{P}, L_{P}\right) & \tau\left(S_{N}, K_{P}, L_{O}\right) \\
\tau\left(S_{N}, K_{O}, L_{N}\right) & \tau\left(S_{N}, K_{O}, L_{P}\right) & \tau\left(S_{N}, K_{P}, L_{O}\right)
\end{array}\right]=\left[\begin{array}{llll}
0.338 & 0.327 & 0.324 \\
0.325 & 0.340 & 0.346 \\
0.330 & 0.327 & 0.324 \\
0.321 & 0.314 & 0.309 \\
0.311 & 0.307 & 0.303 \\
0.303 & 0.318 & 0.314 \\
0.344 & 0.319 & 0.311 \\
0.340 & 0.329 & 0.321 \\
0.351 & 0.396 & 0.333
\end{array}\right]
$$

The matrix of weight impacts of individual states on the output variable calculated on the basis of the defined rules reads:

$$
\rho_{e}(\mathcal{E})=\left[\begin{array}{lll}
\rho\left(S_{O}, K_{N}, L_{N}\right) & \rho\left(S_{O}, K_{N}, L_{P}\right) & \rho\left(S_{O}, K_{N}, L_{O}\right) \\
\rho\left(S_{O}, K_{P}, L_{N}\right) & \rho\left(S_{O}, K_{P}, L_{P}\right) & \rho\left(S_{O}, K_{P}, L_{O}\right) \\
\rho\left(S_{O}, K_{O}, L_{N}\right) & \rho\left(S_{O}, K_{O}, L_{P}\right) & \rho\left(S_{O}, K_{O}, L_{O}\right) \\
\rho\left(S_{P}, K_{N}, L_{N}\right) & \rho\left(S_{P}, K_{N}, L_{P}\right) & \rho\left(S_{P}, K_{N}, L_{O}\right) \\
\rho\left(S_{P}, K_{P}, L_{N}\right) & \rho\left(S_{P}, K_{P}, L_{P}\right) & \rho\left(S_{P}, K_{P}, L_{O}\right) \\
\rho\left(S_{P}, K_{O}, L_{N}\right) & \rho\left(S_{P}, K_{O}, L_{P}\right) & \rho\left(S_{P}, K_{O}, L_{O}\right) \\
\rho\left(S_{N}, K_{N}, L_{N}\right) & \rho\left(S_{N}, K_{N}, L_{P}\right) & \rho\left(S_{N}, K_{N}, L_{O}\right) \\
\rho\left(S_{N}, K_{P}, L_{N}\right) & \rho\left(S_{N}, K_{P}, L_{P}\right) & \rho\left(S_{N}, K_{P}, L_{O}\right) \\
\rho\left(S_{N}, K_{O}, L_{N}\right) & \rho\left(S_{N}, K_{O}, L_{P}\right) & \rho\left(S_{N}, K_{P}, L_{O}\right)
\end{array}\right]=\left[\begin{array}{lll}
-0.34 & -0.02 & 0.26 \\
0.01 & 0.33 & 0.61 \\
0.34 & 0.66 & 0.94 \\
-0.67 & -0.35 & -0.07 \\
-0.32 & 0 & 0.28 \\
0.01 & 0.33 & 0.61 \\
-0.98 & -0.68 & -0.04 \\
-0.65 & -0.33 & -0.07 \\
-0.32 & 0 & 0.28
\end{array}\right] .
$$

Based on the matrix of the calculated values of weighted means and the matrix of weight values of the state of the output variable $e$ of the economic effects of tourism development, defined by ordered triples $\left(S_{i}, K_{j}, L_{k}\right)$, using the Cartesian product $\mathcal{S} \times \mathcal{K} \times \mathcal{L}$ of the family of fuzzy numbers with input variables $x, y, z$ which are classified respectively, and using the following formula:

$$
\chi_{x, y, z}\left(S_{i}, K_{j}, L_{k}\right)=\frac{\rho_{x, y, z}\left(S_{i}, K_{j}, L_{k}\right)}{\tau_{x, y, z}\left(S_{i}, K_{j}, L_{k}\right)}
$$

let us calculate the matrix of characteristic values of output states 


$\chi_{e}(\varepsilon)=\left[\begin{array}{lll}\chi\left(S_{O}, K_{N}, L_{N}\right) & \chi\left(S_{O}, K_{N}, L_{P}\right) & \chi\left(S_{O}, K_{N}, L_{O}\right) \\ \chi\left(S_{O}, K_{P}, L_{N}\right) & \chi\left(S_{O}, K_{P}, L_{P}\right) & \chi\left(S_{O}, K_{P}, L_{O}\right) \\ \chi\left(S_{O}, K_{O}, L_{N}\right) & \chi\left(S_{O}, K_{O}, L_{P}\right) & \chi\left(S_{O}, K_{O}, L_{O}\right) \\ \chi\left(S_{P}, K_{N}, L_{N}\right) & \chi\left(S_{P}, K_{N}, L_{P}\right) & \chi\left(S_{P}, K_{N}, L_{O}\right) \\ \chi\left(S_{P}, K_{P}, L_{N}\right) & \chi\left(S_{P}, K_{P}, L_{P}\right) & \chi\left(S_{P}, K_{P}, L_{O}\right) \\ \chi\left(S_{P}, K_{O}, L_{N}\right) & \chi\left(S_{P}, K_{O}, L_{P}\right) & \chi\left(S_{P}, K_{O}, L_{O}\right) \\ \chi\left(S_{N}, K_{N}, L_{N}\right) & \chi\left(S_{N}, K_{N}, L_{P}\right) & \chi\left(S_{N}, K_{N}, L_{O}\right) \\ \chi\left(S_{N}, K_{P}, L_{N}\right) & \chi\left(S_{N}, K_{P}, L_{P}\right) & \chi\left(S_{N}, K_{P}, L_{O}\right) \\ \chi\left(S_{N}, K_{O}, L_{N}\right) & \chi\left(S_{N}, K_{O}, L_{P}\right) & \chi\left(S_{N}, K_{P}, L_{O}\right)\end{array}\right]=\left[\begin{array}{lll}-0.99 & -0.06 & 0.8 \\ 0.03 & 0.97 & 1.76 \\ 1.03 & 2.01 & 2.9 \\ -2.09 & -1.13 & -0.23 \\ -1.03 & 0 & 0.92 \\ 0.03 & 1.04 & 1.94 \\ -2.85 & -2.13 & -1.3 \\ -1.91 & -1 & 0.22 \\ -0.09 & 0 & 0.84\end{array}\right] \approx\left[\begin{array}{lll}-1 & -0 & 1 \\ 0 & 1 & 2 \\ 1 & 2 & 3 \\ -2 & -1 & -0 \\ -1 & 0 & 1 \\ 0 & 1 & 2 \\ -3 & -2 & -1 \\ -2 & -1 & 0 \\ -0 & 0 & 1\end{array}\right]$.

Comparing the corresponding values of the matrix of the cluster of states $\left[K^{\star}\right]$ and the matrix of base clusters $\left[K^{0}\right]$, let us group the states into base clusters. Thus, the states that belong to the base clusters are the following:

$[-3]^{0}=\left\{\left(S_{N}, K_{N}, L_{N}\right)\right\}$,

$[-2]^{0}=\left\{\left(S_{P}, K_{N}, L_{N}\right),\left(S_{N}, K_{N}, L_{P}\right),\left(S_{N}, K_{P}, L_{N}\right),\right\}$

$[-1]^{0}=\left\{\left(S_{O}, K_{N}, L_{N}\right),\left(S_{P}, K_{N}, L_{P}\right),\left(S_{P}, K_{P}, L_{N}\right)\right.$

, $\left.\left(S_{N}, K_{N}, L_{O}\right),\left(S_{N}, K_{P}, L_{P}\right),\left(S_{N}, K_{O}, L_{N}\right)\right\}$

$[0]^{0}=\left\{\left(S_{O}, K_{N}, L_{P}\right),\left(S_{O}, K_{P}, L_{N}\right),\left(S_{P}, K_{N}, L_{O}\right)\right.$
, $\left.\left(S_{P}, K_{P}, L_{P}\right),\left(S_{P}, K_{O}, L_{N}\right),\left(S_{N}, K_{P}, L_{O}\right),\left(S_{N}, K_{O}, L_{P}\right)\right\}$

$[1]^{0}=\left\{\left(S_{O}, K_{N}, L_{O}\right),\left(S_{O}, K_{P}, L_{P}\right),\left(S_{O}, K_{O}, L_{N}\right)\right.$

, $\left.\left(S_{P}, K_{P}, L_{O}\right),\left(S_{P}, K_{O}, L_{P}\right),\left(S_{N}, K_{P}, L_{O}\right)\right\}$

$[2]^{0}=\left\{\left(S_{O}, K_{P}, L_{O}\right),\left(S_{O}, K_{O}, L_{P}\right),\left(S_{P}, K_{O}, L_{O}\right)\right\}$

$[3]^{0}=\left\{\left(S_{O}, K_{O}, L_{O}\right)\right\}$

From this we can conclude that we have grouped 27 possible evaluations of the value of the output variable into 7 clusters, namely:

$$
\left[K^{0}\right]=\left\{[-3]^{0},[-2]^{0},[-1]^{0},[0]^{0},[1]^{0},[2]^{0},[3]^{0}\right\}
$$

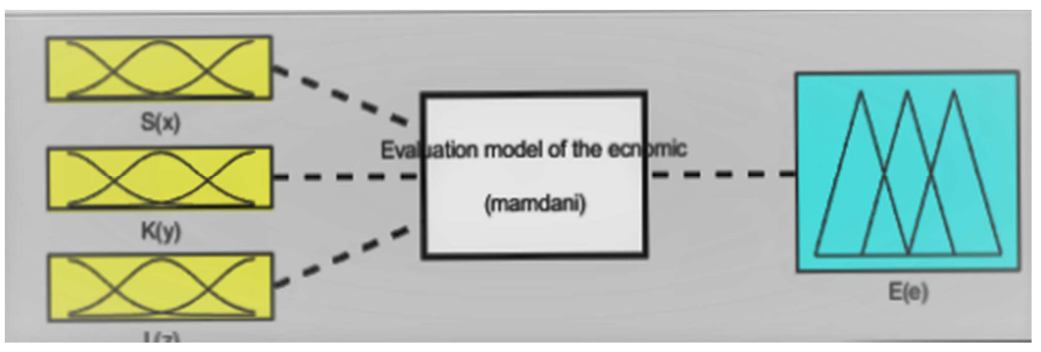

Figure 7. Evaluation model of the economic effects of the impact of tourism developme.

and the coefficient of overnight stays is unsustainable and the

\section{If... Then Rules of Inference}

After modeling the input variables and clustering the values of the output variable, let us apply the if-then rules to linguistic variables $S, K, L$ using the Mammadi's inference rules,(Figure 7$)$, based on the minimum operator $([6,15])$. Let us mark the propositions in the following order

$$
\begin{gathered}
p_{i} \equiv \text { "x is } S_{i} ", q_{j} \equiv \text { "y is } K_{j} ", r_{k} \equiv " z \text { is } L_{k} " \\
e_{n} \equiv \text { "e is } E_{n} "
\end{gathered}
$$

then, the if-then rules can be written in the form of

$$
p_{i} \wedge q_{j} \wedge r_{k} \wedge e_{n}=\min \left(\mu_{S_{i}}(x), \mu_{K_{j}}(y), \mu_{L_{k}}(z), \mu_{E_{n}}(e)\right)
$$

where $e_{n}=e_{i j k}$, and $i, j, k=1,2,3$, and triple of variables $(x, y, z) \subset(\mathcal{S} \times \mathcal{K} \times \mathcal{L}) \subset \mathcal{U}_{1} \times \mathcal{U}_{2} \times \mathcal{U}_{3}$.

Example 3 . Rule $\mathrm{R} 1$ according to our designations reads

$$
p \wedge q \wedge r \wedge e=\min \left(\mu_{S_{N}}(x), \mu_{K_{N}}(y), \mu_{L_{N}}(z), \mu_{E_{A}}(u)\right)
$$

or $\left[\left(x\right.\right.$ is $\left.S_{N}\right)$ and $\left(y\right.$ is $\left.K_{N}\right)$ and $\left(z\right.$ is $\left.\left.L_{N}\right)\right]$ then $e$ is $E_{A}$ which we read as follows:

If the rate of seasonality of overnight stays is unsustainable, impact of tourism development on the local economy is insignificant and declining, the impact of tourism development on the local economy is alarming.

As the output variable of the model is classified by a family of five fuzzy numbers, let us add the state from cluster [-3] to cluster [-2], and state from cluster [3] to cluster [2], then for each fuzzy number from family $\mathcal{E}(e)$ there is a corresponding base cluster with states, as follows:

1. the states from the base cluster

$[-2]^{\prime}=[-2] \cup[-3]$ correspond to a fuzzy number: state is alarming,

2. the states from the base cluster $[-1]$ correspond to a fuzzy number: state is intolerable,

3. the states from the base cluster [0] correspond to a fuzzy number: state is tolerable,

4. the states from the base cluster [1] correspond to a fuzzy number: state is good,

5. the states from the base cluster [2]' $=[2] \cup[3]$ correspond to a fuzzy number: state is sustainable.

We can now define the rules of inference:

1. IF ( $x$ is $S_{N}$ and $y$ is $K_{N}$ and $z$ is $L_{N}$ )

OR ( $x$ is $S_{P}$ and $y$ is $K_{N}$ and $z$ is $L_{N}$ )

$\mathrm{OR}\left(x\right.$ is $S_{N}$ and $y$ is $K_{N}$ and $z$ is $\left.L_{P}\right)$ 
OR ( $x$ is $S_{N}$ and $y$ is $K_{P}$ and $z$ is $L_{N}$ ) THEN $e$ is $E_{A}$

2. IF ( $x$ is $S_{O}$ and $y$ is $K_{N}$ and $z$ is $L_{N}$ )

$\operatorname{OR}\left(x\right.$ is $S_{P}$ and $y$ is $K_{N}$ and $z$ is $\left.L_{P}\right)$

$\operatorname{OR}\left(x\right.$ is $S_{P}$ and $y$ is $K_{P}$ and $z$ is $\left.L_{N}\right)$

OR ( $x$ is $S_{N}$ and $y$ is $K_{N}$ and $z$ is $\left.L_{O}\right)$

$\operatorname{OR}\left(x\right.$ is $S_{N}$ and $y$ is $K_{P}$ and $z$ is $\left.L_{P}\right)$

OR ( $x$ is $S_{N}$ and $y$ is $K_{O}$ and $z$ is $L_{N}$ ) THEN $e$ is $E_{N}$

3. IF ( $x$ is $S_{O}$ and $y$ is $K_{N}$ and $z$ is $L_{P}$ )

$\operatorname{OR}\left(x\right.$ is $S_{O}$ and $y$ is $K_{P}$ and $z$ is $L_{N}$ )

OR ( $x$ is $S_{P}$ and $y$ is $K_{N}$ and $z$ is $L_{O}$ )

OR ( $x$ is $S_{P}$ and $y$ is $K_{P}$ and $z$ is $\left.L_{P}\right)$

$\operatorname{OR}\left(x\right.$ is $S_{P}$ and $y$ is $K_{O}$ and $z$ is $\left.L_{N}\right)$

OR ( $x$ is $S_{N}$ and $y$ is $K_{P}$ and $z$ is $L_{O}$ )

OR ( $x$ is $S_{N}$ and $y$ is $K_{O}$ and $z$ is $L_{P}$ ) THEN $u$ is $E_{P}$

4. IF ( $x$ is $S_{O}$ and $y$ is $K_{N}$ and $z$ is $L_{O}$ )

$\mathrm{OR}\left(x\right.$ is $S_{O}$ and $y$ is $K_{P}$ and $z$ is $L_{P}$ )

OR ( $x$ is $S_{O}$ and $y$ is $K_{O}$ and $z$ is $\left.L_{N}\right)$

OR ( $x$ is $S_{P}$ and $y$ is $K_{P}$ and $z$ is $\left.L_{O}\right)$

OR ( $x$ is $S_{P}$ and $y$ is $K_{O}$ and $z$ is $\left.L_{P}\right)$

OR ( $x$ is $S_{N}$ and $y$ is $K_{P}$ and $z$ is $L_{O}$ ) THEN $e$ is $E_{D}$

5. IF ( $x$ is $S_{O}$ and $y$ is $K_{P}$ and $z$ is $L_{O}$ )

$\operatorname{OR}\left(x\right.$ is $S_{O}$ and $y$ is $K_{O}$ and $z$ is $L_{P}$ )

OR ( $x$ is $S_{P}$ and $y$ is $K_{O}$ and $z$ is $L_{O}$ )

$\operatorname{OR}\left(x\right.$ is $S_{O}$ and $y$ is $K_{O}$ and $z$ is $L_{O}$ ) THEN $e$ is $E_{O}$

\section{Aggregation and Defuzzification}

Let the measured values of the input variable-indicators of the model be the following; $x_{0}, y_{0}, z_{0}$. By coding, we transform the obtained input values into the corresponding values of linguistic variables. The rule

$$
x=x_{0} \in \mathcal{U}_{1}=[0,1], y=y_{0} \in \mathcal{U}_{2}=[0,1],
$$

$z=z_{0} \in \mathcal{U}_{3}=[0,1]$ determines the singleton

$$
\begin{gathered}
; \mu_{S_{i}}\left(x_{0}\right), \mu_{S_{i+1}}\left(x_{0}\right) ; \mu_{K_{j}}\left(y_{0}\right), \mu_{K_{j+1}}\left(y_{0}\right) \\
\mu_{L_{k}}\left(z_{0}\right), \mu_{L_{k+1}}\left(z_{0}\right), i, j, k=1,2,3 .
\end{gathered}
$$

respectively. The strength of the rule is:

$$
\begin{gathered}
\alpha_{i, j, k}=\mu_{S_{i}}\left(x_{0}\right) \wedge \mu_{K_{j}}\left(y_{0}\right) \wedge \mu_{L_{k}}\left(z_{0}\right) \\
\left.=\min \left(\mu_{S_{i}}\left(x_{0}\right), \mu_{K_{j}}\left(y_{0}\right), \mu_{L_{k}}\left(z_{0}\right)\right)\right) \\
\alpha_{i, j, k+1, l+1}=\mu_{S_{i}}\left(x_{0}\right) \wedge \mu_{K_{j}}\left(y_{0}\right) \wedge \mu_{L_{k+1}}\left(z_{0}\right) \\
=\min \left(\mu_{S_{i}}\left(x_{0}\right), \mu_{K_{j}}\left(y_{0}\right), \mu_{L_{k+1}}\left(z_{0}\right)\right) \\
\ldots \ldots \ldots \ldots \\
\alpha_{i+1, j+1, k+1}=\mu_{S_{i+1}}\left(x_{0}\right) \wedge \mu_{K_{j+1}}\left(y_{0}\right) \wedge \mu_{L_{k+1}}\left(z_{0}\right) \\
=\min \left(\mu_{S_{i+1}}\left(x_{0}\right), \mu_{K_{j+1}}\left(y_{0}\right), \mu_{L_{k+1}}\left(z_{0}\right)\right) .
\end{gathered}
$$

For each rule there is one control output variable determined by the conjunction of the strength of the rule and fuzzy sets of the output variable, e.g. for the first case it is

$$
\alpha_{i, j, k} \wedge \mu_{E_{n}}\left(e_{0}\right)=\min \left(\alpha_{i, j, k}, \mu_{E_{n}}\left(e_{0}\right)\right) .
$$

From these control output variables, using the aggregation technique based on the maximum operator, we obtain one output variable.

$$
\begin{gathered}
\mu_{a g g}\left(u_{0}\right)=\max \left\{\alpha_{i, j, k} \wedge \mu_{E_{i, j, k}}\left(e_{0}\right), \alpha_{i, j, k+1} \wedge \mu_{E_{i, j, k+1}}\left(e_{0}\right), \ldots\right. \\
\left.\ldots, \alpha_{i+1, j+1, k+1} \wedge \mu_{E_{i+1, j+1, k+1}}\left(e_{0}\right)\right\} \\
=\max \left\{\min \left(\alpha_{i, j, k}, \mu_{E_{i, j, k}}\left(e_{0}\right)\right), \min \left(\alpha_{i, j, k+1},\right.\right. \\
\left.\left.\mu_{E_{i, j, k+1}}\left(e_{0}\right)\right), \ldots, \min \left(\alpha_{i+1, j+1, k+1}, \mu_{E_{i+1, j+1, k+1}}\left(e_{0}\right)\right)\right\} .
\end{gathered}
$$

In order to obtain a single unique output variable in the form of economic effects of tourism development, it is necessary to complete the defuzzification, i.e. to decode function $\mu_{a g g}(e)$.

There are several methods, and the mostcommonly used one is the gravity center method [13] for continuous fuzzy sets,

$$
e_{E_{n}}^{\star}=\frac{\int e \cdot \sum_{k=1}^{n} \mu_{C_{k}}(e) d e}{\int \sum_{k=1}^{n} \mu_{C_{k}}(e) d e}
$$

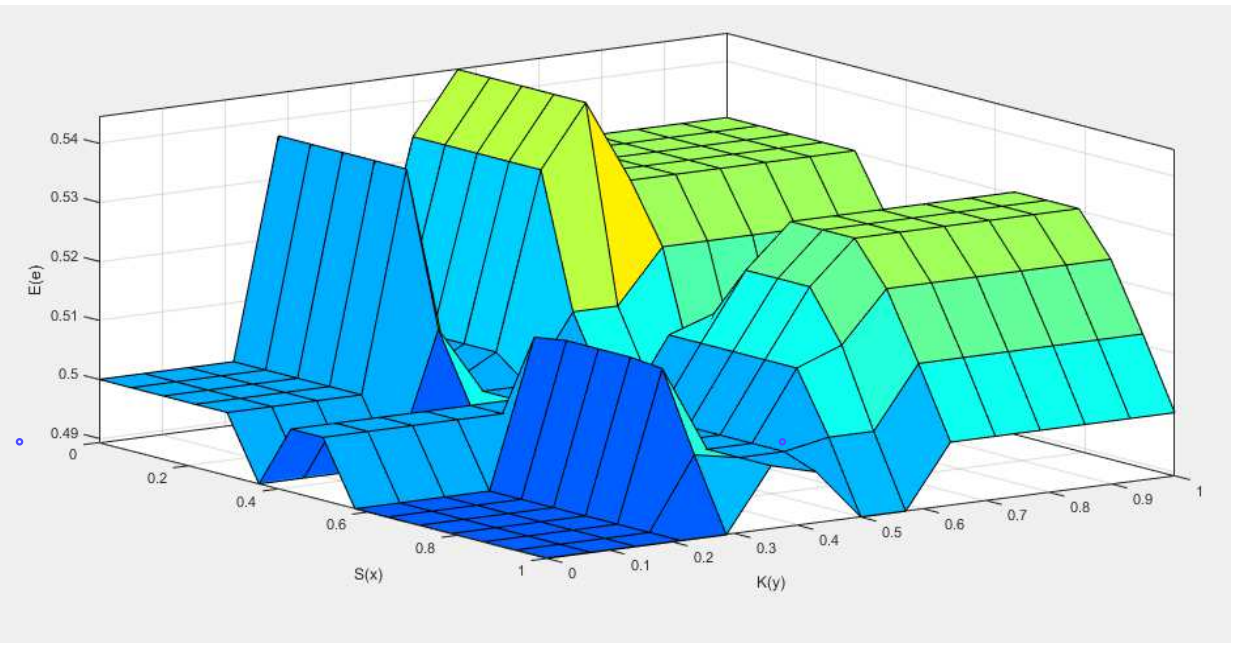

Figure 8. Economic sustainability of tourism development in relation to $S(x)$ and $L(z)$. 


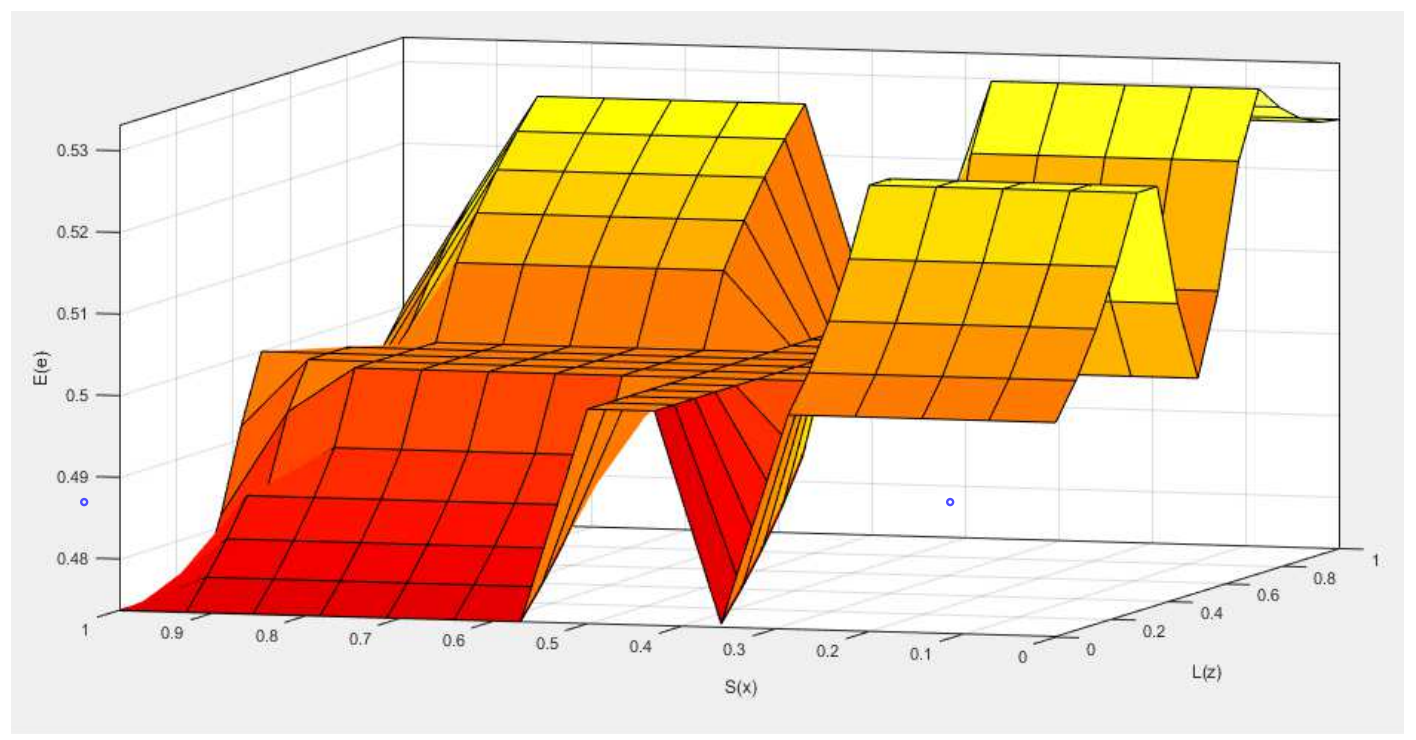

Figure 9. Economic sustainability of tourism development in relation to $S(x)$ and $K(y)$.

Graphic representation of the dependence of economic sustainability of tourism development in relation to the seasonality of visits $S(x)$ and the impact of tourism on the local economy $L(z)$ is shown in Figure 8, and the dependence of economic sustainability on seasonality $S(x)$ and coefficient of the utilization of accomodating capacities $K(y)$ is shown in Figure 9. [15]

\section{Conclusion}

In this paper, we deal in a new way with the problem of grouping fuzzy attributes that describe the state of input values in the fuzzy model. It is known that complex systems described by fuzzy relations generate a large number of inference rules, which is a major problem in determining the unique value of the output variable. In order to reduce the fuzzy rules we group descriptive states of input values into clusters according to certain rules that usually rely on expert experience and acquired knowledge. Ideally, the number of clusters would correspond to the number of attributes by which the value of the output variable is classified, which is not the case. In the absence of an expert, grouping should be realized on the basis of some criteria. One way of grouping descriptive states that define the input values in the fuzzy model into clusters is presented in this paper. The constructed model is based on the interval of dominant affiliation of the variable to the fuzzy set by which the input variable was evaluated, and on the values of the characteristic function defined on the interval of dominant affiliation and on the weight impact of the fuzzy number in the quantification of the input variable. They represent the basis for quantification of the defined characteristic value of the output variable descriptive state in the fuzzy model. For the presented method, the mathematical logical argumentation of the application is given, as well as the algorithm of the constructed method application. The presented grouping algorithm facilitates the formulation of conclusions based on the reduced number of fuzzy rules generated by the attributes for estimating the values of input variables in the fuzzy model and provides the possibility to conclude about the output variable attribute value, in the absence of an expert, based on the quantitative value of characteristic elements of input variables. The conclusion we derive from such an algorithm is based on quantitative indicators of the input fuzzy numbers in the model and does not require the presence of an expert. The method can be applied very easily in cases when it is possible to compensate for the descriptive states of the input values when estimating the output variable. This compensation is based on equal values of characteristic functions of input fuzzy attributes, which describe the situation, and on the basis of which the unique characteristic value of the fuzzy model output variable attributes is determined, which is shown in the example of assessing the economic sustainability of tourism development based on the comparative sustainability indicators.

\section{References}

[1] Jovicic, D., Turizam i zivotna sredina-koncepcija odrzivog turizma, Zaduzbina Andrijevic, Beograd. (2000).

[2] Pap, E., Fazi mjere i njihova primjena, Univerzitet u Novom Sadu, PMF, Novi Sad. (1999).

[3] Teodorovic, D., Kikuchi, S.: Uvod u teoriju fuzzy skupova $i$ primjene u saobracaju, Saobracajni fakultet $\mathrm{u}$ Beogradu. (1994).

[4] Ma, Z.; Zhang, F.; Yan, L.; Cheng, J., Fuzzy Knowledge Management for the Semantic Web, XI, 275 p. 67 illus, Hardcover, Springer-Verlag Berlin Heidelberg, (2014).

[5] Zadeh, L. A., The concept of a lingustic variable and its application to aproximate reasoning, American Elsevire Publishing Company, (1973).

[6] Mamdani, H. E., Application of fuzzy logic to approximative reasoning using linguistic systems, IEEE Transc Computer,(26) pp. 1189-1191. (1987). 
[7] Kovacic, Z., Bogdan, S. Inteligentno upravljanje sustavima, Fakultet elektrotehnike i racunarstva, Univerzitet u Zagrebu, Zagreb, (2000).

[8] Hans, M.; Applied fuzzy aritmetic-an intraduction with engineering applications, Springer, (2005).

[9] Stojanovic, N., Primjena teorije fazi skupova na odredjivanje inteziteta održivog razvoja turizma u zaštićenim područjima, PMF, Novi Sad, Disertacija. (2007).

[10] Stojanovic, N., Mathematical modling with fuzzy sets of sustainable tourism development, Interdisciplinary Description of Complex Systems 9 (2), 134-160, (2011).

[11] Stojanovic, N.: Application of Fuzzy Logic in Modeling Market Brand Value. American Journal of Mathematical and
Computer Modelling. Vol. 4, No. 1. pp. 1-15.(2019). doi: 10.11648/j.ajmcm.20190401.11

[12] Stojanovic, N.,: Measurung sustainability of touri sm development-Aplication of fuzzy logic, LAP Lambert, Academic Publishing, Saarbrucken, Germany, (2015).

[13] Cupic, M., Basic,. D., B., Golub, M.: Neizrazito, evolucijsko i neuroracunarstvo. Zagreb. (2013).

[14] Braee, M., Rutherford, A. D.: Theoretical and Linguistic Aspects of the Fuzzy Logic Controller, Automatica. Vol. 15. pp. 553-577. (1979).

[15] N. S. Sivanandam, N. S., Sumathi, S. Deepa, S. N., Introducion to Fuzzy Logic using MATLAB, Springer, Verlag Berlin Heidelberg. (2007). 